\title{
Proposition pour le déchiffrement de l'élamite linéaire
} Matthieu Kervran

Cet article présente la démarche menant au déchiffrement de l'élamite linéaire. Cette écriture étant représentée par peu de textes, des doutes subsistent sur la valeur de certains signes, et des erreurs sont sans doute présentes dans ce travail. Afin de faciliter la critique et la correction de ces erreurs, la démarche est donnée ici dans l'ordre chronologique qui a été suivi. Les hypothèses testées et rejetées sont cependant omises.

Dans un premier temps, nous nous limiterons aux textes des vases gunagi en argent, puis nous tenterons d'appliquer les résultats issus de ce corpus aux inscriptions sur les autres objets.

Les translitérations adoptées sont celles de [EW] (qa dans [EW] au lieu de $k a_{4}$ dans [EKI], etc.). Toutes les séquences en élamite linéaire ont été orientées de gauche à droite pour faciliter la lecture, quel que soit leur orientation originelle, sauf indication contraire.

Dans les références bibliographiques, le nombre qui suit la référence est le numéro de page, sauf pour $[\mathrm{EKI}]$ où c'est le numéro du texte et du paragraphe.

Toutes les images des signes et des textes proviennent du corpus présenté dans [Desset 2018], sauf A' et W qui proviennent de [Mäder et al. 2017]. Les rares divergences sur la représentation graphique des textes sont notées en rouge. Les images tirées d'autres ouvrages sont indiquées le cas échéant.

Les résultats de cet article ont été appliqués à l'étude du proto-élamite. Cette étude fait l'objet d'un second article, qui confirme une partie des résultats obtenus ici, permet de déchiffrer certains signes syllabiques du proto-élamite, et affine le déchiffrement de l'élamite linéaire. 


\section{Première étape : Bala-iššan, « lani šarih » et Idaddu}

\section{Bala-iššan}

[Desset 2018] a repéré les noms de Šilhaha et Ebarat dans les textes X, H', J' et F'. Si on fait l'hypothèse que le signe (et sa variante $九$ dans H', I' et K') représente le mot $\grave{u}$ « je », on peut penser que les mots qui suivent ce signe sont des noms de souverains. En particulier dans K', qui est un texte utilisant le séparateur de mots, on peut trouver la séquence $C+D X+1 *$, qui est un bon candidat comme nom propre (cf. [Desset 2018:136]). Avec les valeurs postulées dans cet article, on lit $b(a)$-?- $N A P I^{I}-(u) \breve{s}-\check{s} a$-in. A condition de supposer que $\oslash$ ne soit pas un déterminatif mais un signe phonétique, on peut faire correspondre cette séquence avec le nom de Bala-iššan ${ }^{2}$, en posant $\theta=i$ et $\uparrow=l a$. Ceci reste cohérent avec la lecture classique du nom d'Insušinak dans la séquence

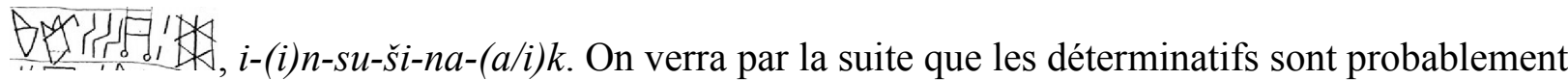
totalement absents en élamite linéaire.

\section{Lani šarih}

La séquence $\left.\right|^{\mid{ }_{\text {mIII }}}$ se retrouve, en général au début d'une ligne, sur Q, W, X, Y, Z, A', F', H', I', J' et

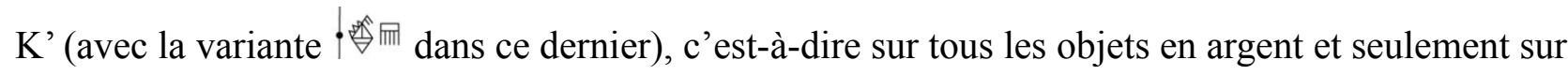
ceux-là, à l'exception de L' qui est aussi l'inscription la plus courte, et sans doute un faux (voir la partie « Authenticité de L'»).

De plus, ${ }^{, \pi ⿵ 冂 卄}$ s'insère dans une séquence plus longue :

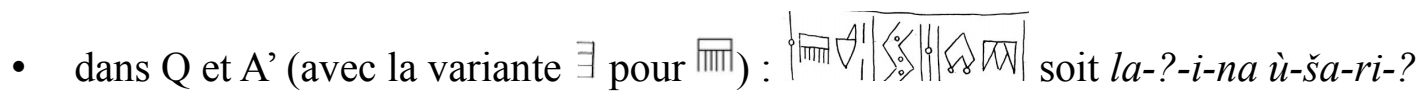

- dans X et J' : 俩监网 soit la-?-ša-(i)r-?

- dans F' et $\mathrm{K}^{\prime}$ : $^{\text {|⿴囗十ा }}$

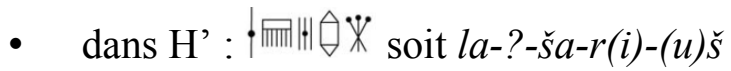

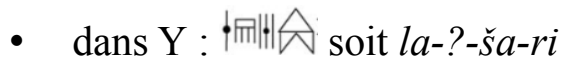

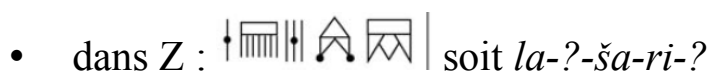

Les textes utilisant des séparateurs de mots (Q, F', K', Z) nous assurent que cette séquence forme

1 Déterminatif des noms divins.

2 Écrit ainsi dans [EW:131]. On utilise de préférence la transcription des textes élamites plutôt qu'akkadiens, et si possible de la période des sukkalmah.

3 On verra par la suite que la différence entre $\hat{\theta}$ et $\hat{\theta}$ est réelle. 
une unité. Il a déjà été noté dans [Mäder et al. 2017:34] que 㒳 ih/išs (d'après l'article cité) et $\mathbb{X}(u) \check{s}$ pouvaient être des suffixes verbaux, $-\check{s}$ représentant la 3ème personne du singulier. Vu le contexte (présence de $\grave{u}$ « je » dans Q et $\mathrm{A}^{\prime}$ ), on peut reprendre l'idée que 㒳 $=-h^{4}$, et représente ici la lère personne du singulier.

Or le mot ša-ri-h est connu ([EW:1137]) et traduit «j'ai fondu/fait fondre », précédé par des noms de métaux (la-an-si-ti-ir-ra ir ša-ri-h « en or je l'ai fondu/fait fondre » dans [EKI 10a IV, 10b IV, 10c IV] ; zu-ba-ar la-an-si-ti-in-ni ša-ri-h « en bronze et en or j’ai fondu/fait fondre » dans [EKI 46 $\S 12]$ et suivants, [EKI 47 §9] entre autres).

Lani étant le mot élamite pour « argent », on peut poser mint $=n i$. La variante la-ni-i-na dans Q, A' et $\mathrm{W}$ peut être une forme adjectivale, voir la-ni-i-ni « en argent» ([EW:816]).

\section{Idaddu}

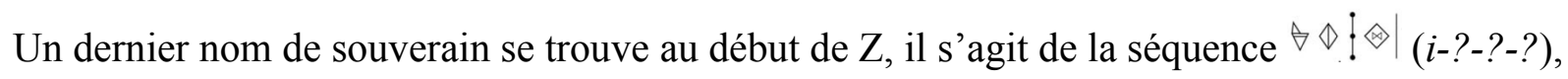
qu'on retrouve aussi dans $\mathrm{Y}$, dans le texte qui est à côté de la jambe du personnage agenouillé. Le seul souverain connu dont le nom corresponde est Idaddu (écrit $i$-da-at-du ou $i$-ta-at-du selon les textes). On pose donc $\downarrow=t a / d a, \vdots=-t$ et $\otimes=t u / d u$.

D'autre part, il est préférable de lire le nom d'Ebarat tel qu'il est écrit dans les textes élamites, c'est-

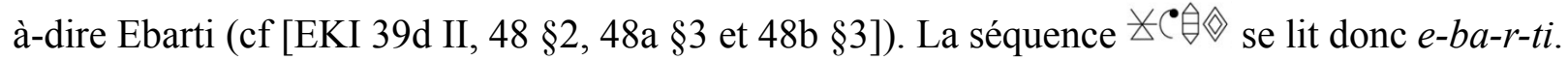




\section{Résultats intermédiaires (première étape)}

$\mathrm{Ci}$-dessous un tableau résumant les signes connus après le déchiffrement des noms d'Insušinak, Napiriša, Ebarti, Šilhaha, Bala-iššan ainsi que de l'expression lani šarih :

\begin{tabular}{|c|c|c|c|c|}
\hline \multirow[t]{2}{*}{ 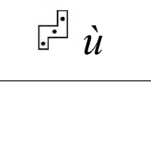 } & $\delta_{i}$ & $\forall_{e}$ & & \\
\hline & $p i$ & & $C_{b a}$ & \\
\hline \multirow[t]{2}{*}{$\otimes t u / d u$} & $\Delta t i$ & & $\downarrow_{t a / d a}$ & $\vdots-t$ \\
\hline & & & & 舟 $-k$ \\
\hline \multirow[t]{5}{*}{$\langle\backslash\rangle_{s u}$} & 月ši & & ||$_{.} \check{s} a$ & $X-\check{S}$ \\
\hline & $\mathbb{1 \pi n t}^{5} n i$ & & $n a$ & 变 $-n$ \\
\hline & & & la & 金 $-l$ \\
\hline & $\widehat{A} r i \ominus r i$ & & & $\hat{\theta}-r$ \\
\hline & & & ha & 网- $h$ \\
\hline
\end{tabular}

Figure 1 : équivalences entre signes élamite linéaire et translittérations du cunéiforme élamite 


\section{Deuxième étape : la titulature}

Etant donné que les textes comportent tous des noms de souverains, les passages qui suivent leur nom et précèdent le passage « lani šarih... » peuvent contenir une liste de leurs titres. Nous allons comparer la lecture des signes connus aux titulatures des textes royaux rassemblées dans [EKI] $p$. 37 note 3 .

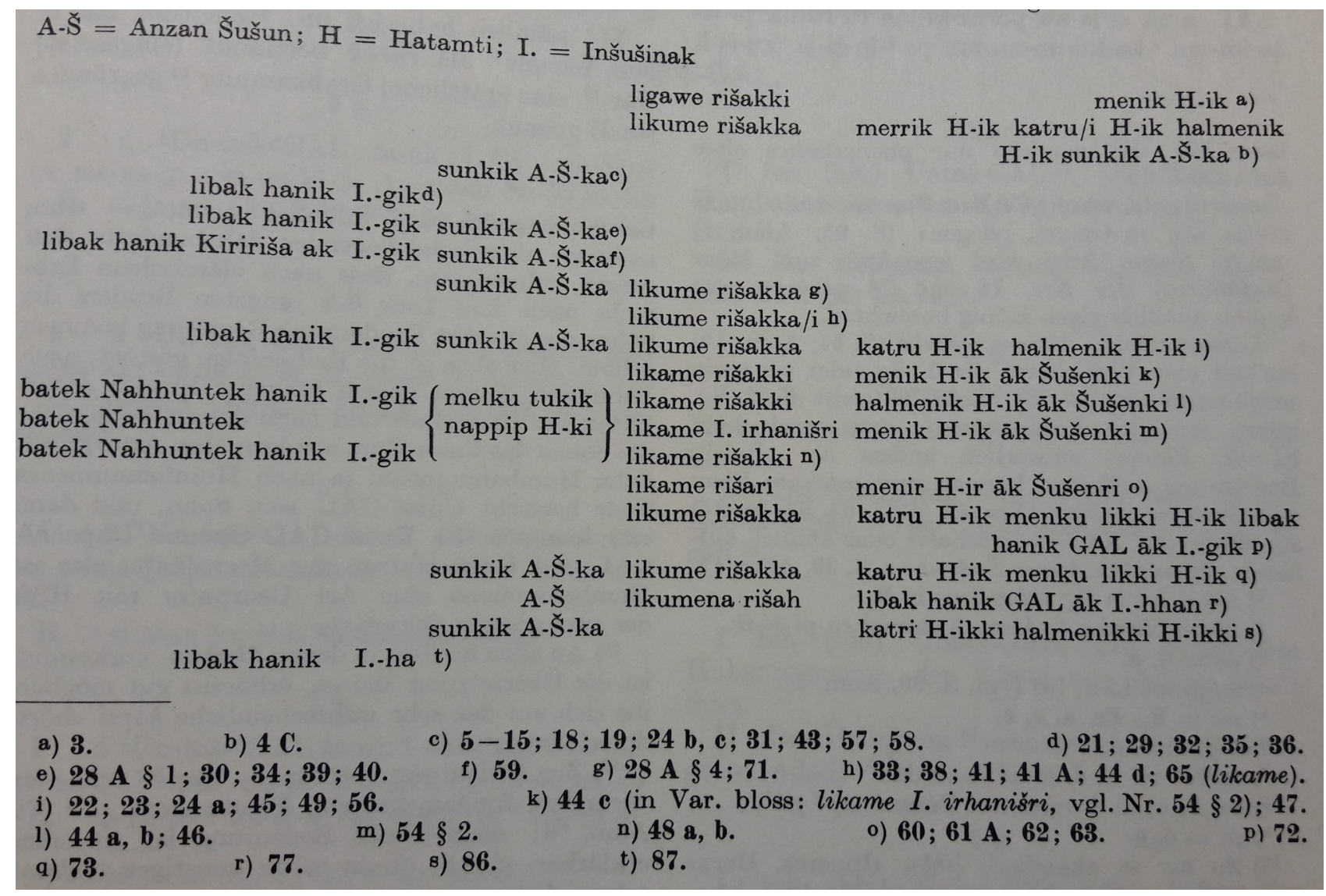

figure 2 : liste des titulatures dans [EKI] p. 37 note 3.

Ci-dessous la lecture que nous pouvons faire des textes avec les signes déjà connus :

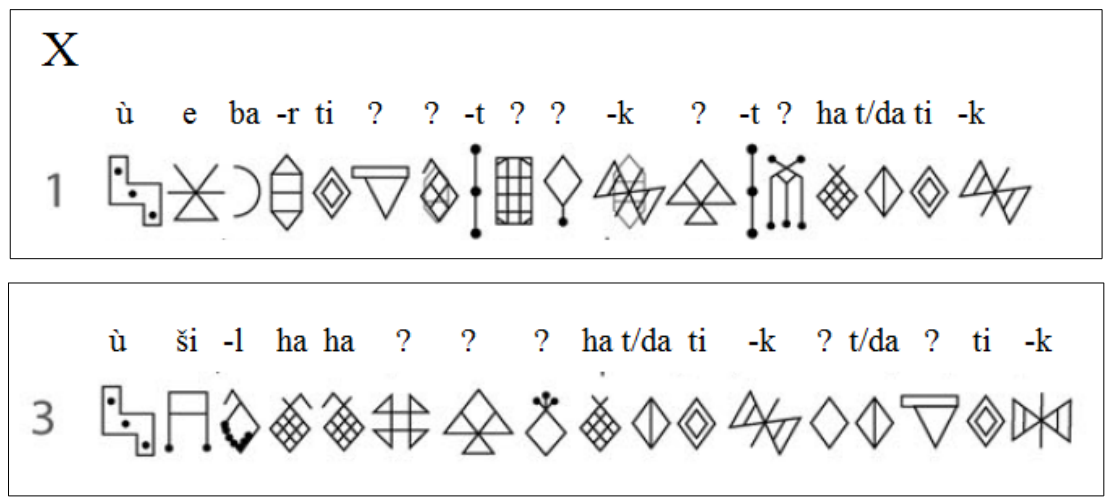


Y ? ? ? ? ? ? ? ? ? ni ? ? ? ? ? - - t ? ?

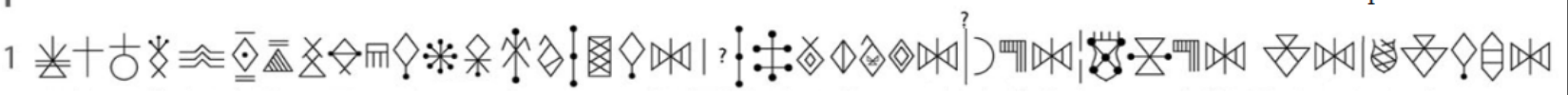

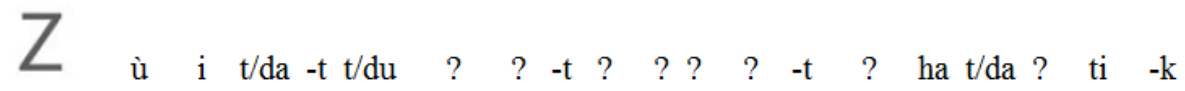

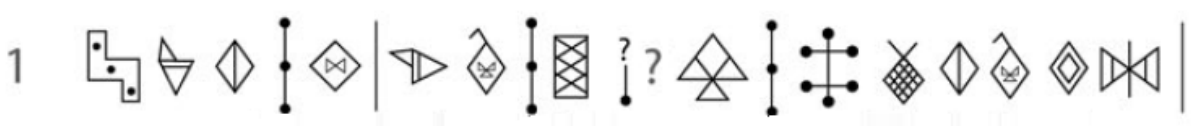 ba ?

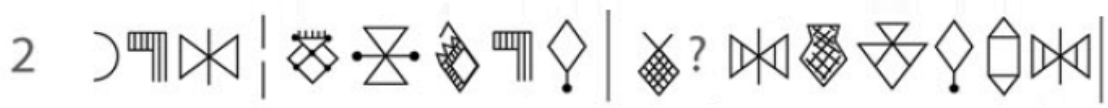

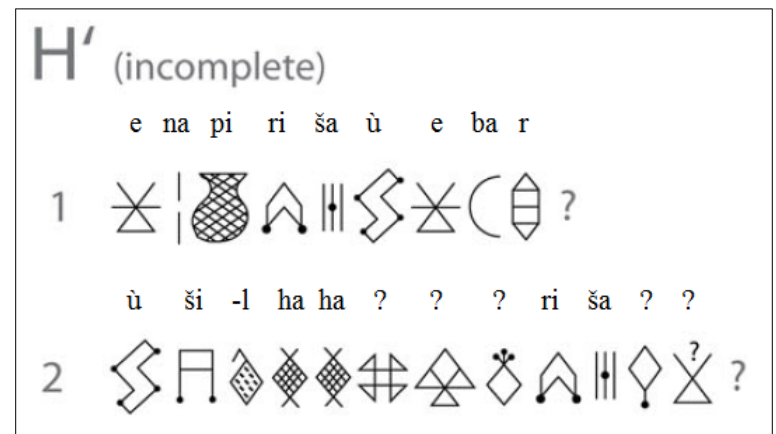

$F^{\prime}$ e ba -r ti ? ?

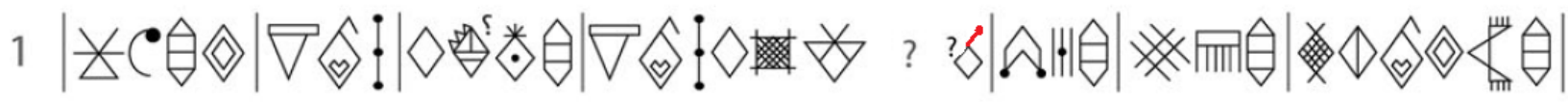

I' (incomplete)

1

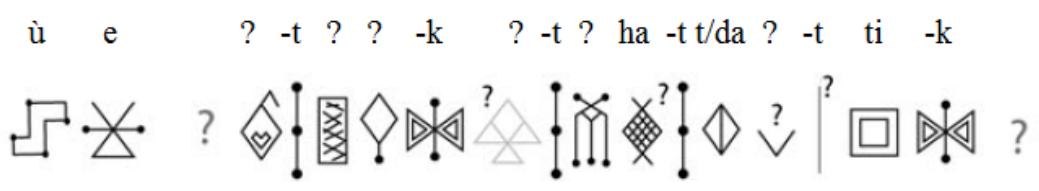
? - - n ni $-\mathrm{r}$ ha $-\mathrm{t} \mathrm{t} / \mathrm{da}$ ? $-\mathrm{t}$ ti $-\mathrm{r}$

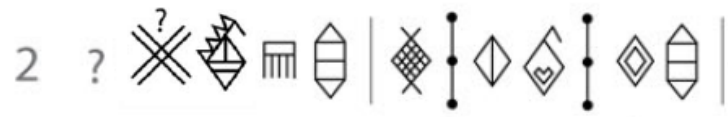



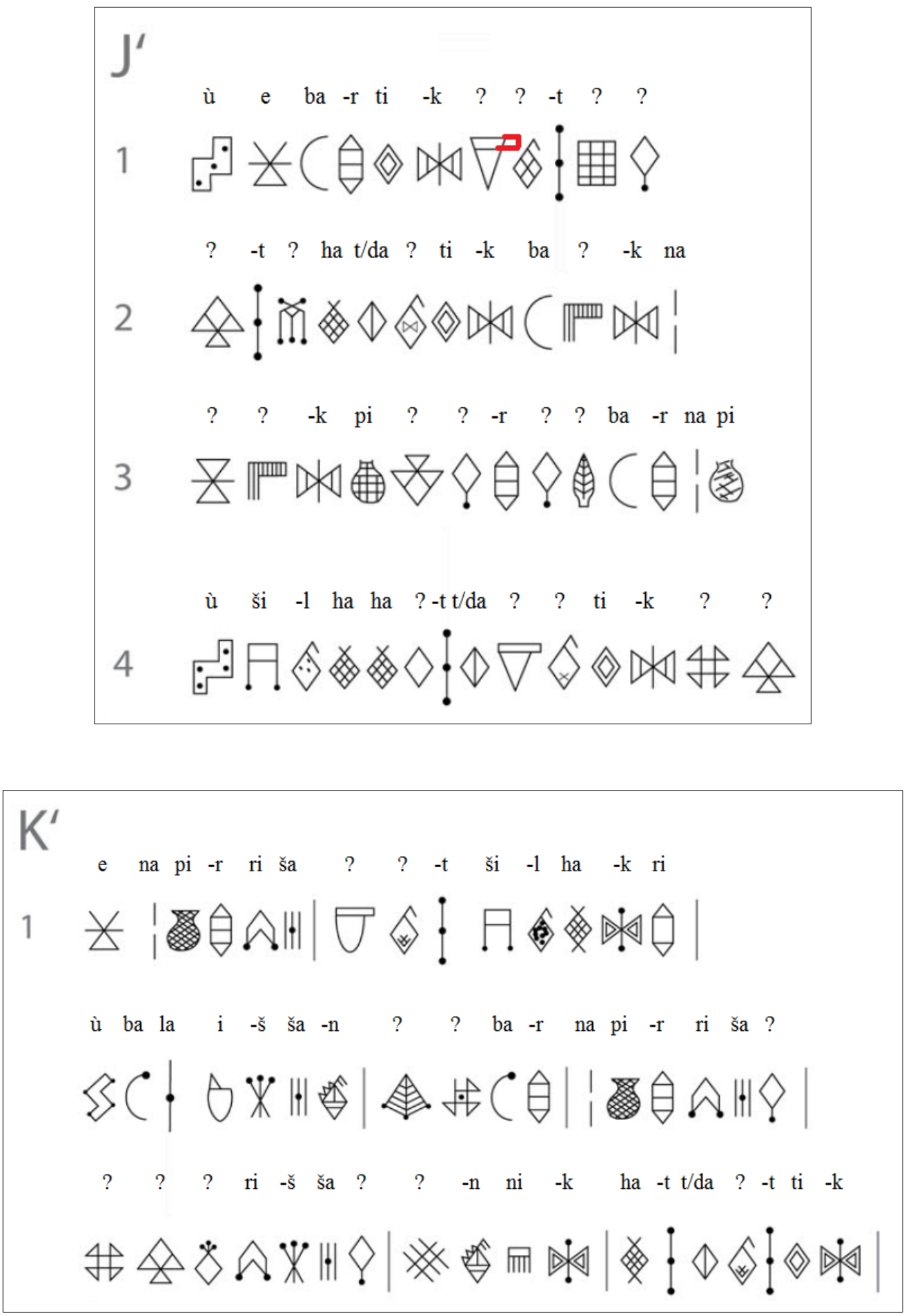

Il est facile de reconnaître le mot hatamtik/hatamtir dans les séquences $h a-(t)-d a-?-(t)-t i-k / h a-(t)-$ $d a-?-(t)-t i-r$. Par Conséquent, $\widehat{\otimes}=-m$ (ainsi que ses variantes graphiques $\hat{\otimes}_{\mathrm{et}} \hat{\otimes}^{\hat{\Delta}}$ ).

On peut aussi reconnaître menik hatamtik / menir hatamtir dans les séquences ?-n-ni-k hatamtik $\left(\mathrm{K}^{\prime}\right)$ et ?-(n)-ni-r hatamtir (F', I'), donc $=m e$.

De même pour qatru hatamtik dans la séquence alternative, ?-t-? hatamtik (X, Y, Z, I', J'). On en 


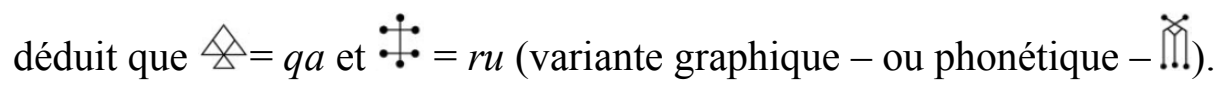

La séquence $\nabla \hat{\otimes} !(?-m-t)$ devient reconnaissable en tant que temt (écrit en général te-im-ti dans les inscriptions cunéiformes ${ }^{6}$. Donc $\nabla=t e$.

On retrouve aussi une séquence commune dans $\mathrm{Y}, \mathrm{Z}$ et J', dans les trois cas après qatru hatamtik:
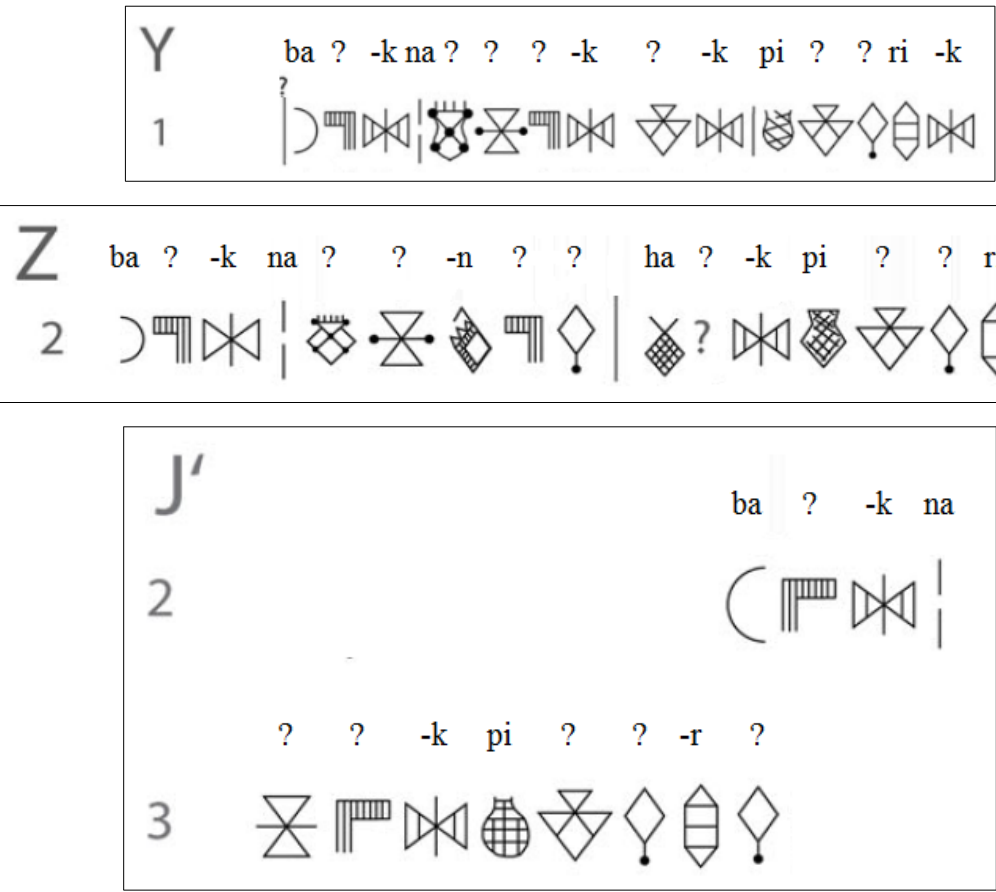

Cela correspond, à quelques adaptations près, à batek Nahhuntek hanik Pinikirki (sur le modèle de hanik Insušnakki qu'on trouve dans EKI 44, 46, 48 entre autres). Le caractère manquant entre ha et $-k$ dans $\mathrm{Z}$ est probablement $\forall$. De même dans Y, il y a un espace important entre $\mathfrak{A}^{\circ}$ et $\forall$. Il est probable qu'un caractère ait été effacé à cet endroit.

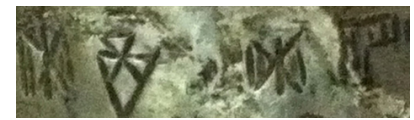

Figure 3 : Y ligne 1. Au centre on voit un espace où un caractère a pu être effacé.

On peut donc poser $\|^{\| m}=t e, \bigotimes=-h, Z=h u, \forall=n i, \hat{Y}=k i$. Comme on sait par ailleurs ([EW:206] bi-ne-en-gi-ir et [EW:617] ha-ni-ěs, et [Tavernier 2007:272]) que Pinikir se prononçait sans doute /pinekir/ et hani-/hane-/, on peut poser que $\forall=/$ ne/ ce qui le différencie de minl/ni/. La lecture des séquences est donc :

Y : ba-te-k na-h-hu-te-k [ha]-ne-k pi-ne-ki-ri-k « berger de Nahhunte, aimé de Pinekir » $\mathrm{Z}:$ ba-te-k na-h-hu-n-te-ki ha-[ne]-k pi-ne-ki-ri-k « berger de Nahhunte, aimé de Pinekir » J' : ba-te-kna-hu-te-k pi-ne-ki-r-ki « berger de Nahhunte (et) Pinekir » 
Ensuite, il reste deux séquences dans ces passages dont la lecture peut se déduire des caractères déjà connus et de la comparaison avec les titulatures courantes :

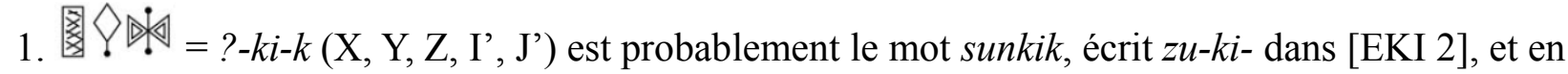
général $s u$-un- $k i$ - dans les textes postérieurs. Donc 图 $=s u / z u$.

2. 将 $\otimes \otimes=?-q a-?$, dans X (?-qa-? ha-t/da-ti-k), Y (i ?-qa-? i-t/da-t/du-ri, à côté de la jambe du personnage agenouillé), H' (?-qa-? ri-ša-ki...), J' (?-qa-[?]), K' (?-qa-? ri-š-ša-ki). La lecture qui s'impose est liqam/we, écrit li-ga-we dans [EKI 3] et li-qa-me ou li-ku-me dans les textes postérieurs. Donc $\mathscr{\sharp}=l i$ et $\mho^{\prime}=m e / w e^{7}$.

Enfin, deux séquences peuvent se lire par comparaison avec d'autres textes :

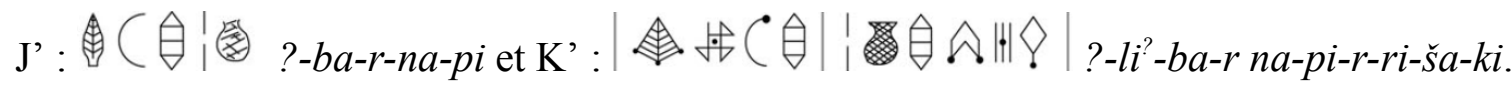

On peut faire l'hypothèse que :

- est une variante graphique de et se lit $l i$ (il y a donc deux caractères pour $l i$, de même qu'il y a deux caractères en élamite linéaire pour $r i, p i, n i$, etc. en cunéiforme - l'un des deux se lit probablement /re/, /pe/, /ne/);

- la ligne 3 de J' est incomplète (comme chaque ligne de ce texte) et doit être complétée en $l i$ ba-r na-pi[-ri-ša-ki] sur le modèle de $\mathrm{K}^{\prime}$;

- l'expression li-ba-r na-pi-ri-ša-ki signifie « (moi) serviteur de Napiriša », sur le modèle $l i$ ba-ar.ma-za-at « serviteur de Manzat » ([EW:818]);

- le caractère $\stackrel{4}{\dagger}$ dans $\mathrm{K}$ ' est soit la consonne isolée $-b$, auquel cas le point central distinguerait ce caractère de $4 t i$, soit une erreur ou un repentir du scribe hésitant entre deux caractères de phonétisme proche, auquel cas le point serait une variante graphique ou un signe de correction, et le repentir expliquerait l'aspect inachevé du signe. Je penche vers la deuxième possibilité, car la consonne isolée - $p$ est identifiée par la suite, et rien ne laisse penser que l'élamite distinguait une série en $b$ et une série en $p$.

7 L'équivalence avec we dans [EKI 3] (alors que takme, šutme et šatme sont attestés tels quels dans ce texte), ainsi que les autres équivalences de ce signe (voir plus bas) montre que le son était sans doute différent de celui de me. Comme c'est ce dernier signe, et pas $\diamond$ qui est utilisé comme suffixe dans les mots abstraits, cela conforte l'interprétation selon laquelle liqam/we désigne la personne et non le territoire. Ainsi i liqam/we Idaddu-ri dans Y signifie « ceci (est) le gouverneur Idaddu » et désigne le personnage représenté, et liqam/we rišakki signifie simplement « le grand gouverneur » et pas « l'agrandisseur du royaume». 


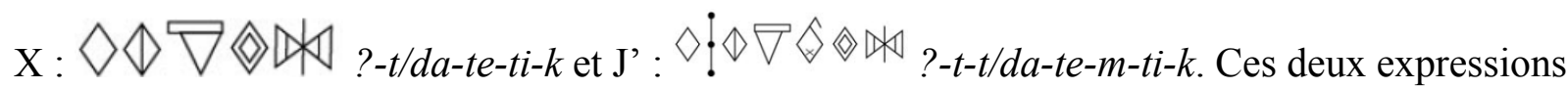
figurent dans la titulature de Šilhaha, dans des textes où figure aussi Ebarti. Or le cylindroïde d'Atta-hušu (voir [MDP 28:7] pour l'original) présente ces deux souverains de la façon suivante : « Ebarat étant roi d'Anšan et de Suse, Šilhaha étant sukkalmah et roi-père (AD.DA LU[GA]L) d'Anšan et de Suse... » ([Sollberger \& Kupper 1971: 260, IVO6a.] réf à vérifier) On peut poser $\diamond=a$ et ainsi établir le parallélisme $a-t-t / d a-t e-m-t i-k=A D \cdot D A L U[G A] L, a d-d a$ signifiant « père » en élamite comme en sumérien. On a aussi accessoirement les équivalences : temt $=$ lugal et likam $/ w e=$ sukkal, et likawe riša $=$ sukkal-mah $($ ou peut-être likam $/ w e=$ sukkal-mah et likawe riša = grand sukkal-mah).

\section{Résultats intermédiaires (deuxième étape)}

\begin{tabular}{|c|c|c|c|c|}
\hline \multirow[t]{2}{*}{$\dot{v}$} & $\vartheta_{i}$ & $\forall_{e}$ & $\nabla_{a}$ & \\
\hline & \multicolumn{2}{|c|}{$p i$} & $C_{b a}$ & \\
\hline \multirow[t]{2}{*}{$\Leftrightarrow t u / d u$} & $\otimes t i$ & $\nabla t e^{8},\|\|^{m} t e$ & $\downarrow_{t a / d a}$ & $\vdots-t$ \\
\hline & \multicolumn{2}{|c|}{$\vartheta_{k i}$} & $\Delta_{q a}$ & 舫 $-k$ \\
\hline\langle\rangle$_{s u}$ & \multicolumn{2}{|c|}{$日_{s ̌ i}$} & $|+|_{\check{s} a}$ & $\Psi_{-\check{s}}$ \\
\hline \multicolumn{5}{|l|}{ 图 $z u / s u$} \\
\hline & & me & & $\hat{\otimes}-m$ \\
\hline & $\mathrm{nin}^{9} n i$ & $\nabla_{n i(/ n e /)}$ & $n a$ & $\otimes^{n}-n$ \\
\hline & \multicolumn{2}{|c|}{ 壮 $l i$, $l i$} & la & 金 $-l$ \\
\hline$\because{ }^{10} r u$ & \multicolumn{2}{|c|}{$\hat{\Delta} r i, \theta r i$} & & $\hat{\theta}-r$ \\
\hline \multirow[t]{2}{*}{$\nabla_{h u}$} & & & $h a$ & 肉 $-h, \otimes-h$ \\
\hline & & $\bigotimes_{w e / m e}$ & & \\
\hline
\end{tabular}

Figure 4 : équivalences entre élamite linéaire et valeurs de l'élamite cunéiforme.

A ce stade, on voit que l'élamite linéaire ne différencie pas entre consonnes sourdes et sonores. On utilisera donc de préférence la consonne sourde (ta pour ta/da, etc.) par la suite. De plus comme l'élamite ne semble pas utiliser d'occlusive uvulaire, on régularisera qa en $k a$.

8 La syllabe notée par $\nabla$ est probablement distincte de celle notée par $\|^{m}$.

9 Variante graphique: $\exists$

10 Variante graphique : I. 
Transcription, translittération et proposition de traduction des titulatures :

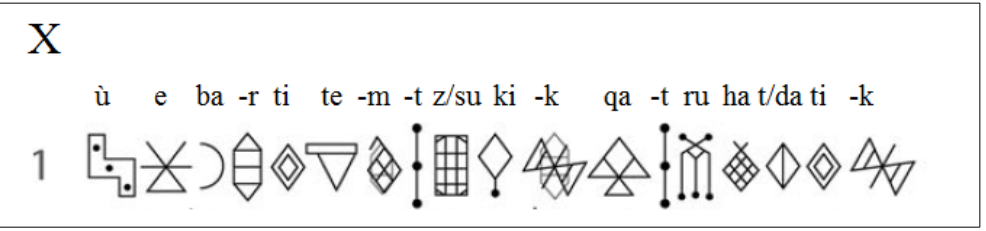

ù Eparti temt zukik katru hata $<m>$ tik

Moi Eparti, seigneur-roi, prince de Hatamti (ou hatamtite)

ù ši -1 ha ha li qa w/me ha t/da ti $-\mathrm{k}$ a t/da te ti $-\mathrm{k}$

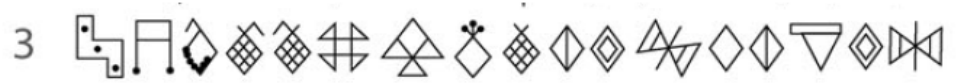

ù Šlhaha likawe hata $<m>$ tik $a<t>$ ta $t e<m>t i k$

Moi Šilhaha, sukkal de Hatamti, père-roi

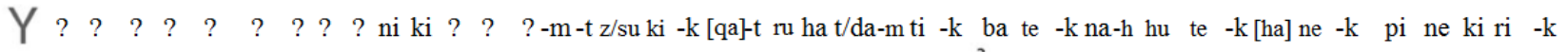

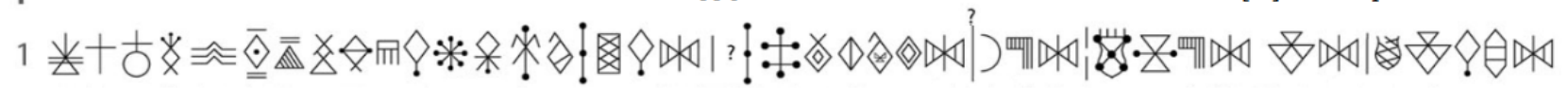
???????"1 <te>mt zukik [ka]tru hatamtik batek nahhu<n>tek [ha]nek pinekirik

??????? seigneur-roi, prince de Hatamti, berger de Nahhunte, aimé de Pinekir

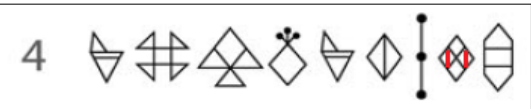

i likawe Itatturi

Ceci (est) le sukkal Idaddu

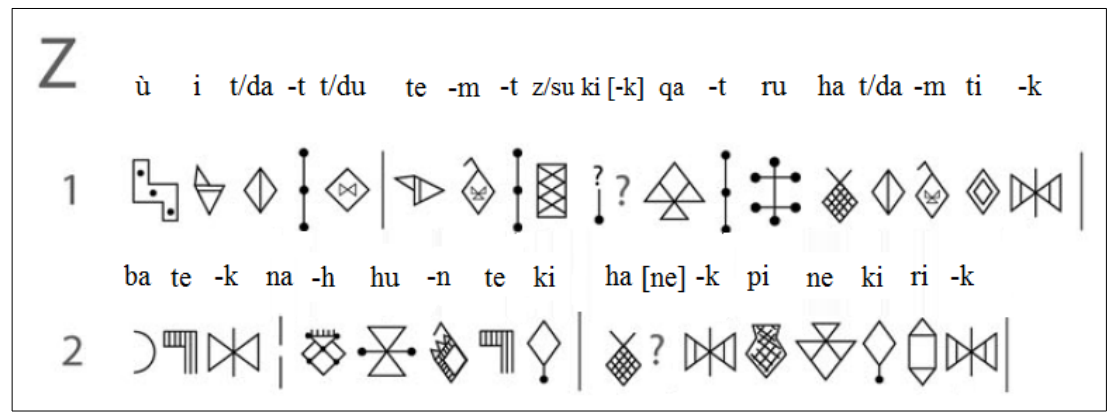

ù Itattu temt zuki[k] katru hatamtik

batek Nahhunteki ha[ne]k pinekirik

Moi Idaddu, seigneur-roi, prince de Hatamti,

berger de Nahhunte, aimé de Pinekir

11 Le début de Y est mystérieux, constitué de hapax, puis de quelques caractères non superposables sur un texte connu. Le texte semble redevenir compréhensible au deuxième caractère du mot temt. 


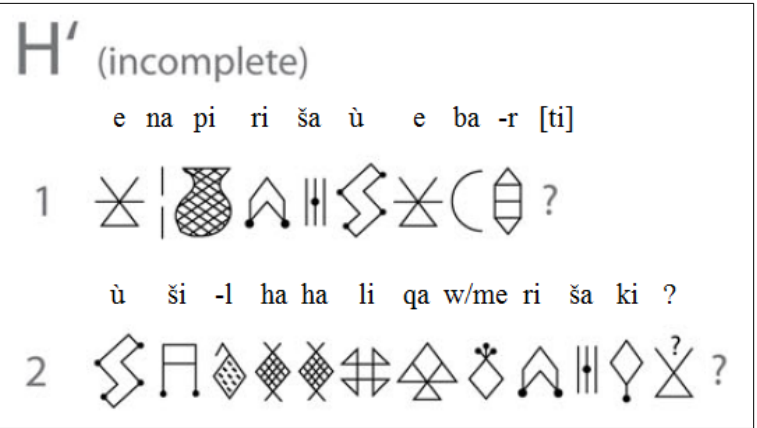

e Napiriša ù Epar[ti] [...]

ù Šilhaha likawe rišaki [...]

Ô Napiriša, moi Eparti, [...]

Moi Silhaha, sukkal-mah, [...]

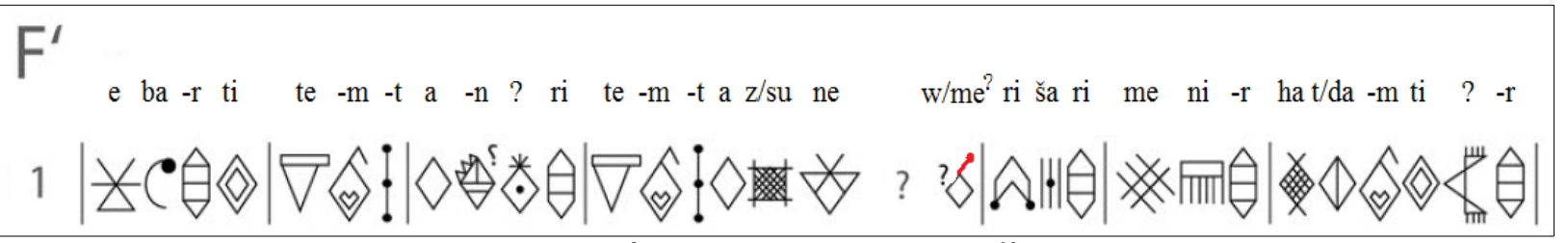

Eparti temt an? ri temt azune? [lika]we? rišari menir hatamti? ${ }^{12} r$

Eparti, seigneur?, seigneur?, sukkal-mah?, dirigeant des? Hatamti

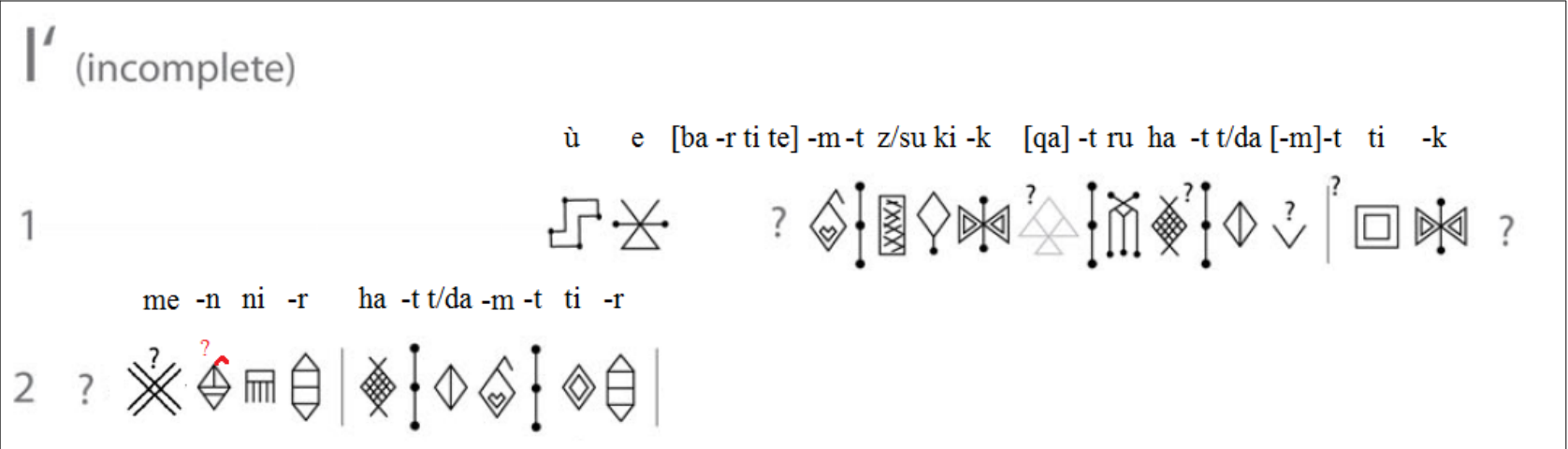

ù E[parti te]mt zukik [ka]tru hatta[m]ttik?

? mennir hattamttir

Moi Eparti, seigneur-roi, prince de Hatamti ?

? ( $3^{\text {ème }}$ pers.) dirigeant de Hatamti

12 Voir dans [Mäder et al. 2017:35] la proposition $\$$ m $=p i$ (marque du pluriel). Le pluriel peut être lié aux deux occurences de temt $X$ qui précèdent. 


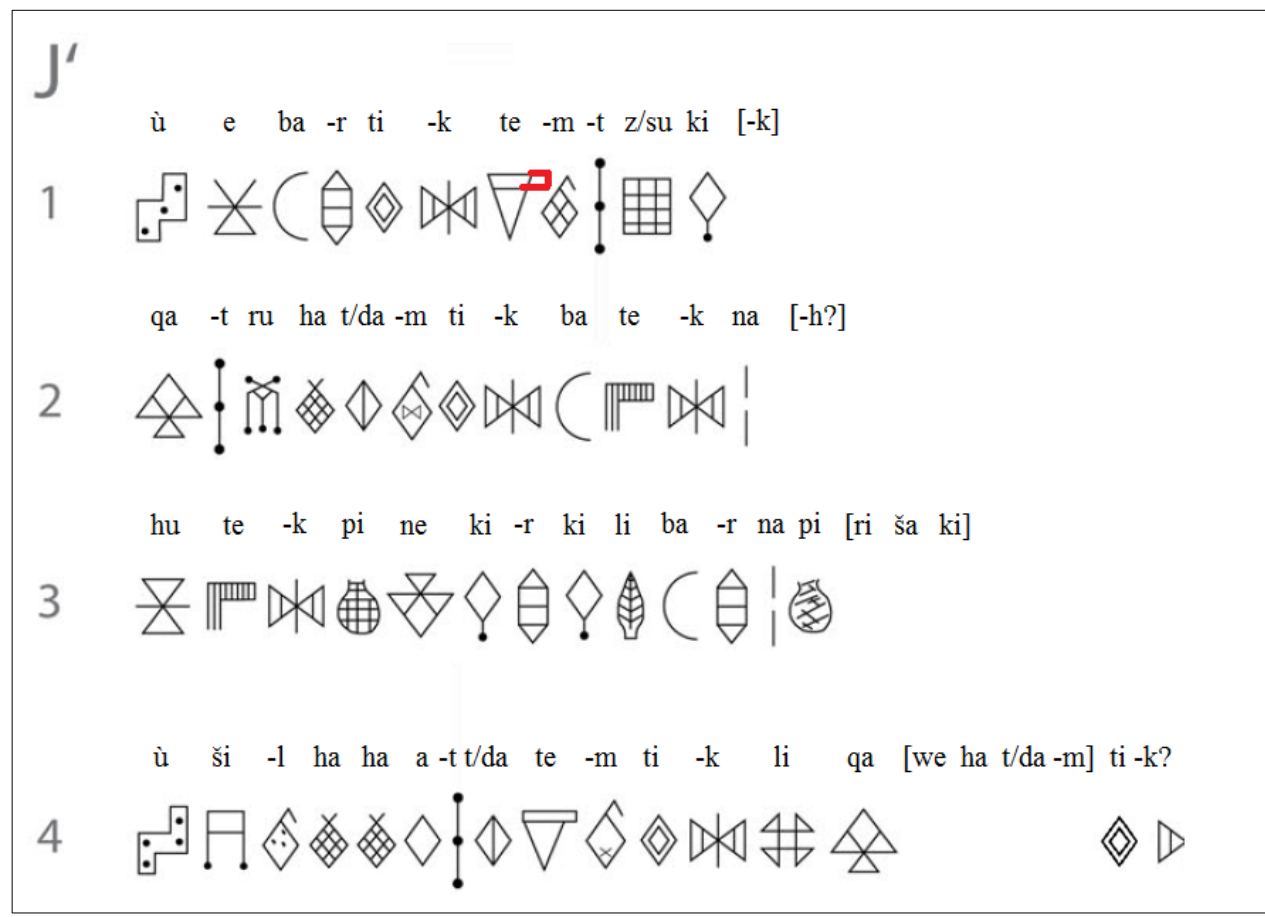

ù Epartik temt zuki [k]

katru hatamtik batek $\mathrm{Na}[\mathrm{h}-]$

-hutek pinekirki libar Napi[rišaki]

ù Šlhaha atta temtik lika[we Hatam] tik ${ }^{\text {?I3 }}$

Moi Eparti, seigneur-roi

prince de Hatamti, berger de

Nahhunte et Pinekir, serviteur de Napiriša

Moi Šilhaha, père-roi, suk[kal de Hatam]ti

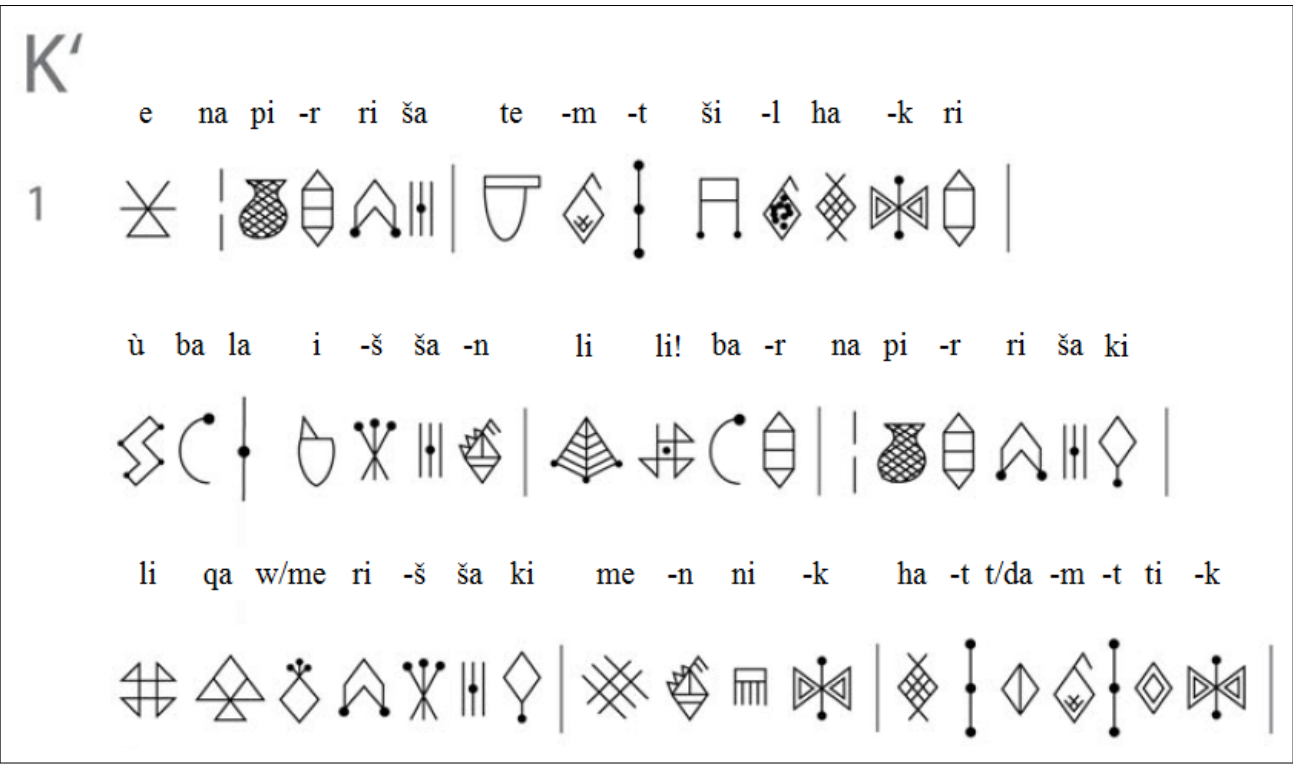

13 Il est certain, par comparaison avec les autres textes, qu'une partie du texte de J' a été effacée à la droite de l'inscription. Les lignes 4 et 5 se poursuivent après la partie effacée. On peut supposer que $\diamond \triangleright$ est la suite logique de la ligne $4(\operatorname{cf} X)$, et que $\triangleright$ est ici la partie gauche d'un $\$ partiellement effacé. L'espace vide dans la ligne est cohérent avec les caractères supposés. 
e Napirriša temt šilhakri

ù Pala-iššan lipar Napirrišaki

likawe riššaki mennik Hattamttik

Ô Napiriša, le seigneur fort?

Moi Pala-iššan, serviteur de Napiriša,

sukkal-mah, dirigeant de Hatamti

\section{Troisième étape : le reste des textes des gunagi}

Nous pouvons maintenant tenter de lire les textes en intégralité et chercher des correspondances dans les textes royaux pour déchiffrer plus de signes.

Nous reprenons à notre compte la proposition classique $D O=p u$, qui permet de lire hahpuh/hahpuš «j'ai/il a écouté » (dans X, Z, H'), ainsi que le nom de Puzur-Šušinak dans les textes de Suse.

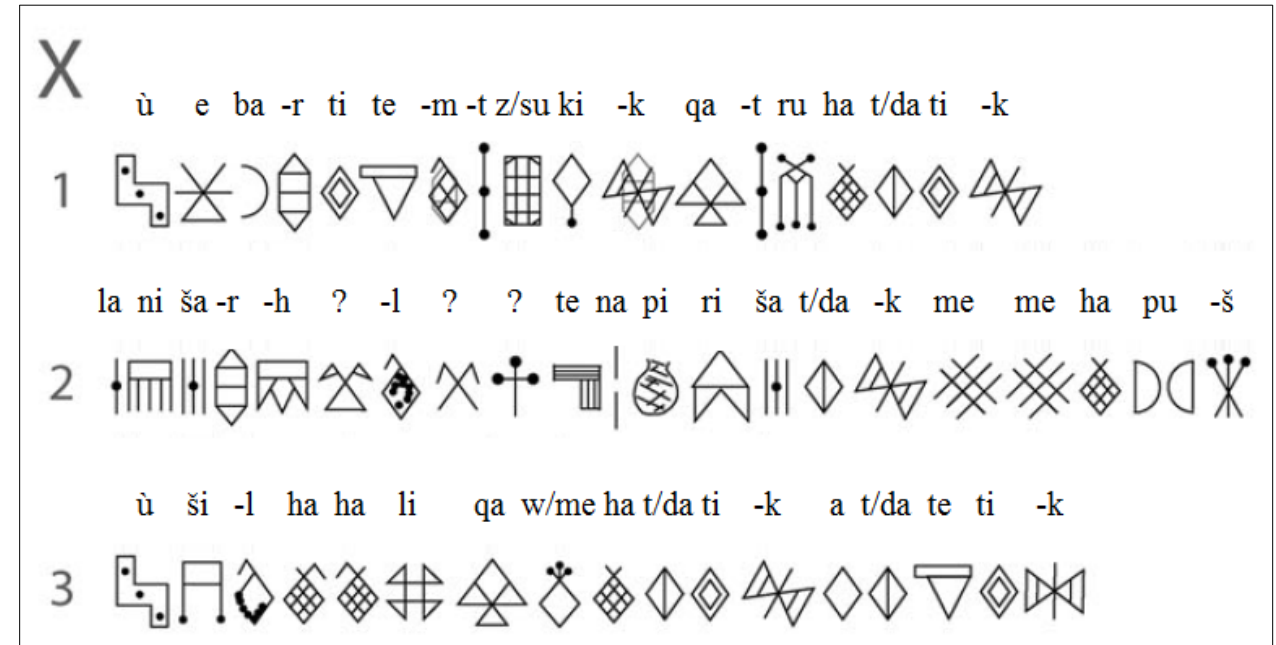

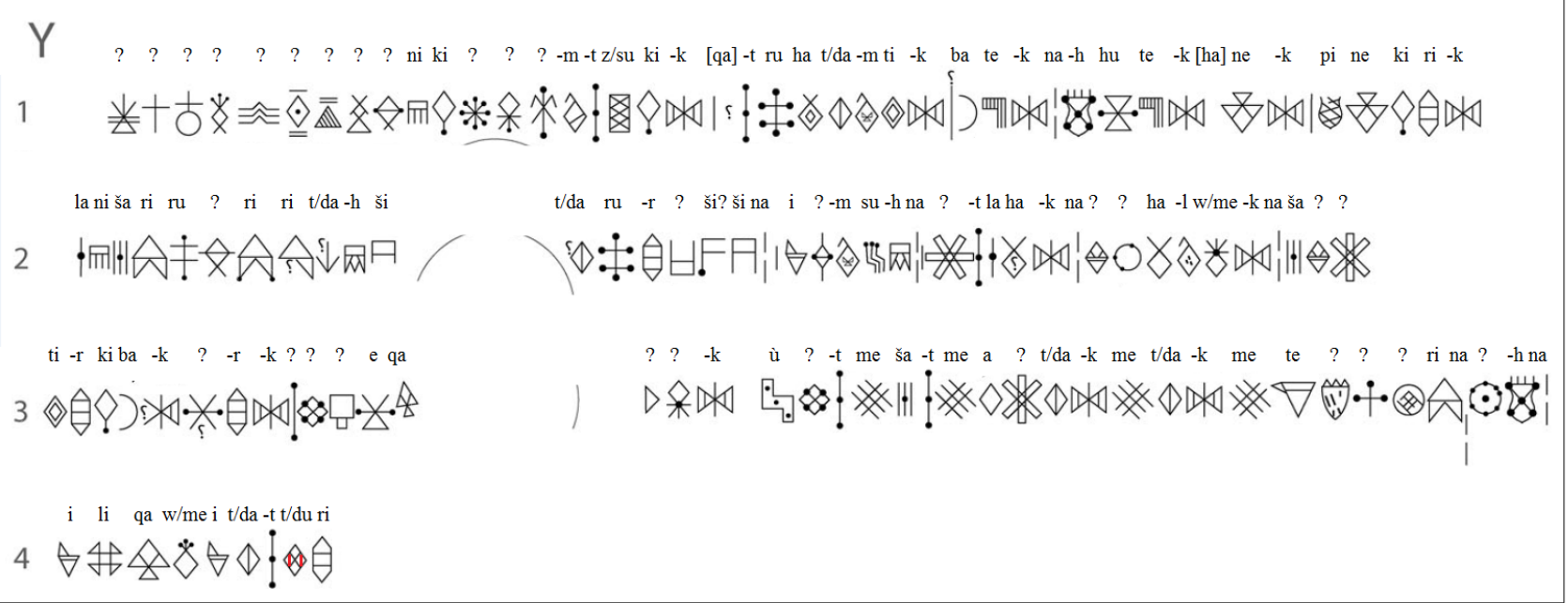




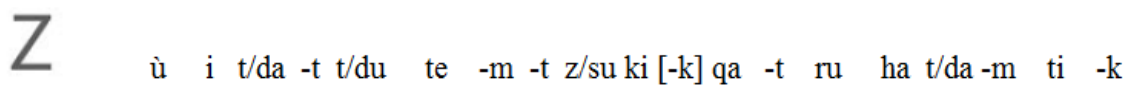

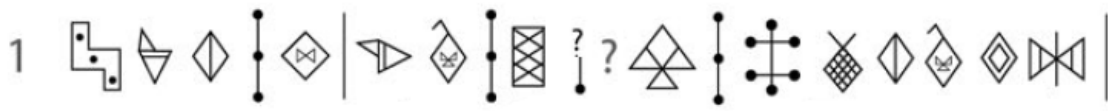
ba te $-\mathrm{k}$ na $-\mathrm{h}$ hu $-\mathrm{n}$ te $\mathrm{ki}$ ha [ne]-k pi ne ki ri $-\mathrm{k}$

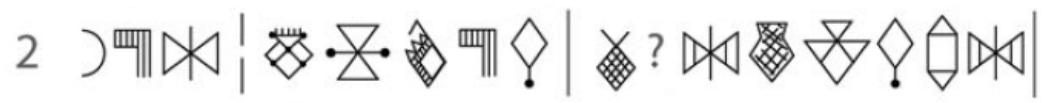
$\mathrm{t} / \mathrm{da} \quad-\mathrm{k}$ me ha $-\mathrm{h}[\mathrm{pu}]$-h $\quad$ ?

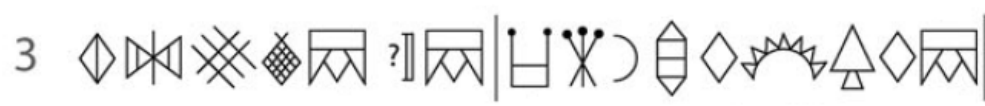
la ni ša ri $-\mathrm{h}$ na pi ri ša ? te hu ? ša $-\mathrm{k}$ ki $-\mathrm{r}$

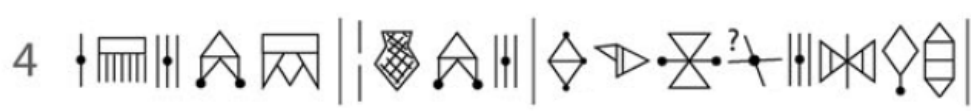
? ? ri ? t/da -h ? ? te -n ti ? -t la ha -k na

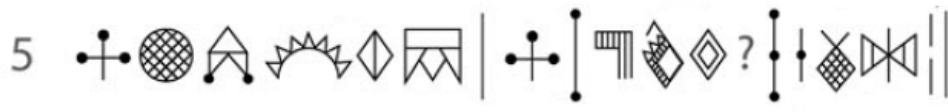
? ? ha $-1 \mathrm{w} / \mathrm{me}-\mathrm{k}$ na ša ? ? ti ? ki ba $-\mathrm{k}$ na

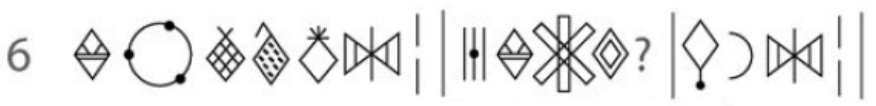
$\mathrm{z} / \mathrm{su}-\mathrm{n} \mathrm{z} / \mathrm{su}-\mathrm{n} \quad$ ?

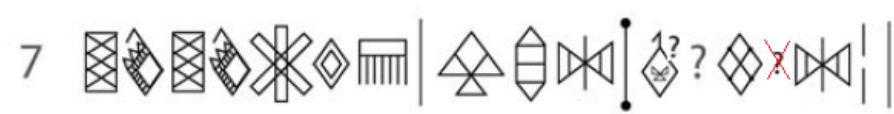

e qa ? ? na w/me ni ? ? ? ? hu

$8 \forall \cdot \Delta \Delta ?$ ? ? - $\mathrm{t} \mathrm{t} / \mathrm{da}-\mathrm{k}$ ? me ša $-\mathrm{t}$ me $\mathrm{t} / \mathrm{da}-\mathrm{k}$ me te ? i ? ha $-\mathrm{h}$ pu ?

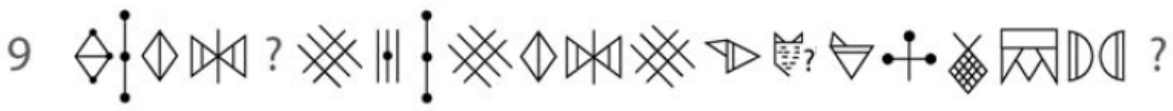




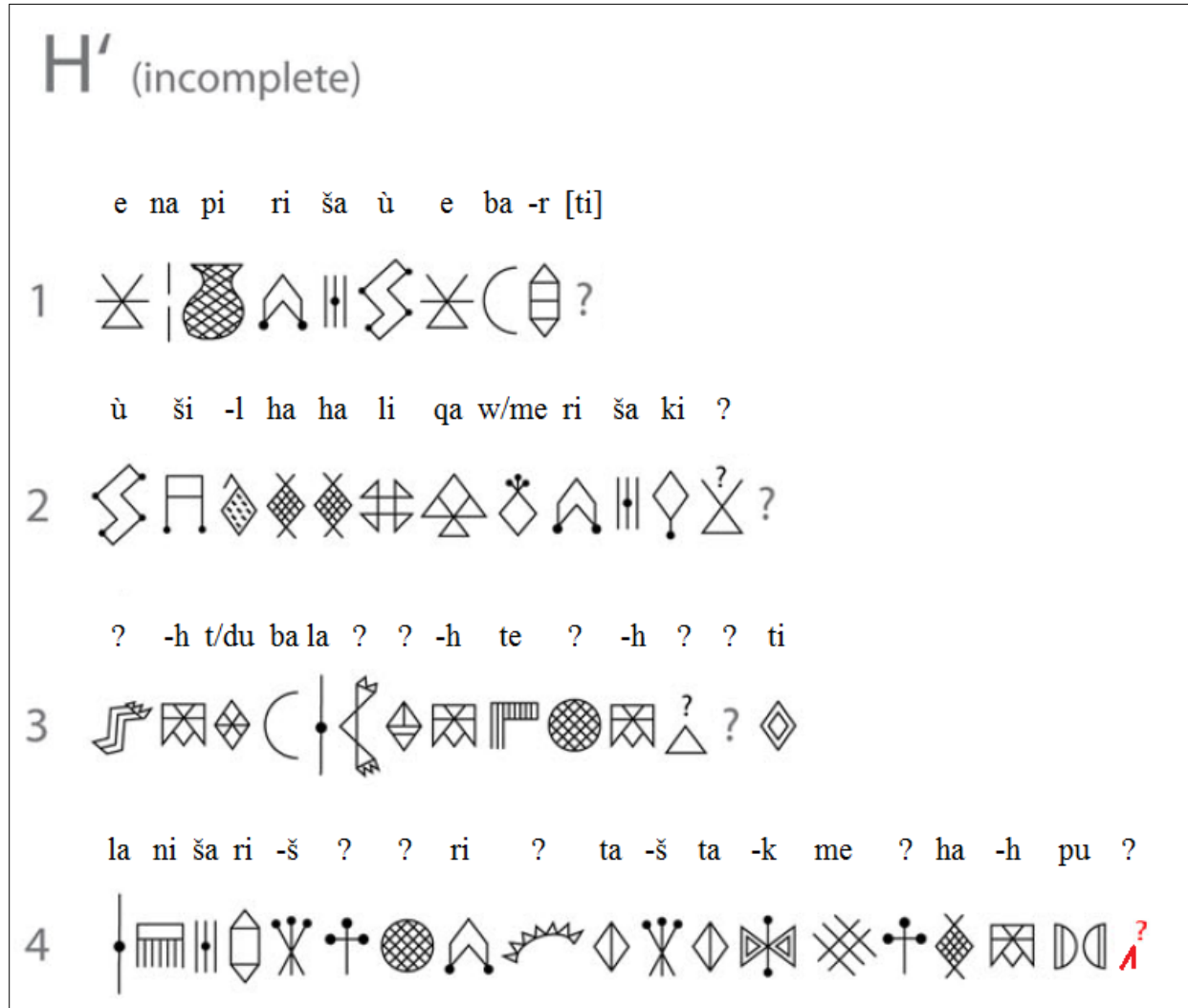

$F^{\prime}$ e ba -r ti te $-m$-t a $-n$ ? ri te $-m$-t a $z / s u$ ne $\quad w / m e^{?}$ ri ša ri me ni $-r$ ha t/da $-m$ ti $\quad$ ? $-r$

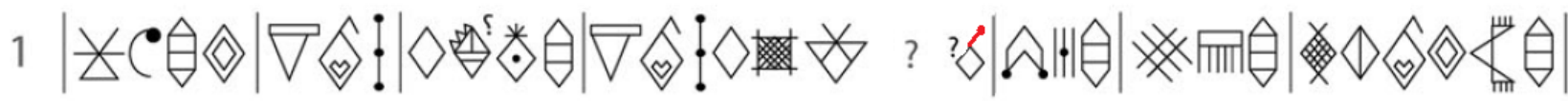
la ni ša ri - h na pi ri ša $w / m e$ ? ha la ?

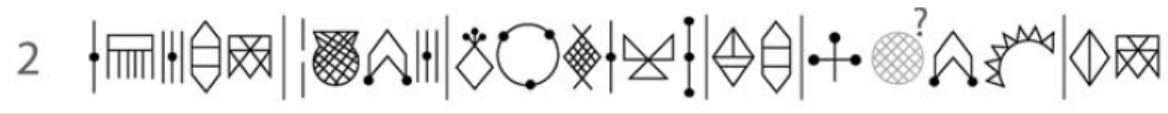

I' (incomplete)

1 me -n ni -r ha -t t/da -m -t ti - r la ni

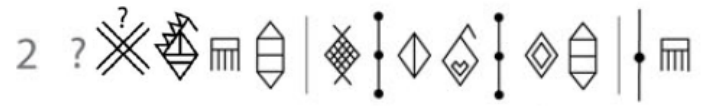

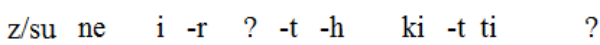

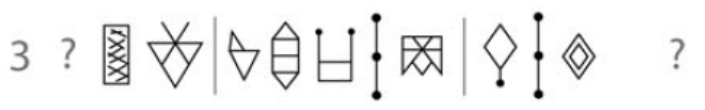

ù e [ba-r ti te] -m -t z/suki -k qa -t ru ha -t t/da [-m-t] ti $-k$

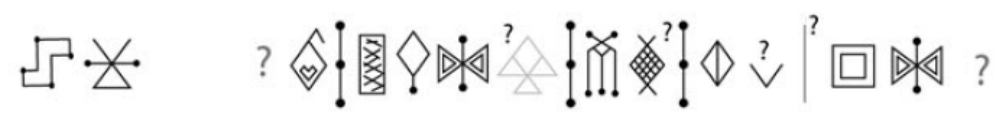




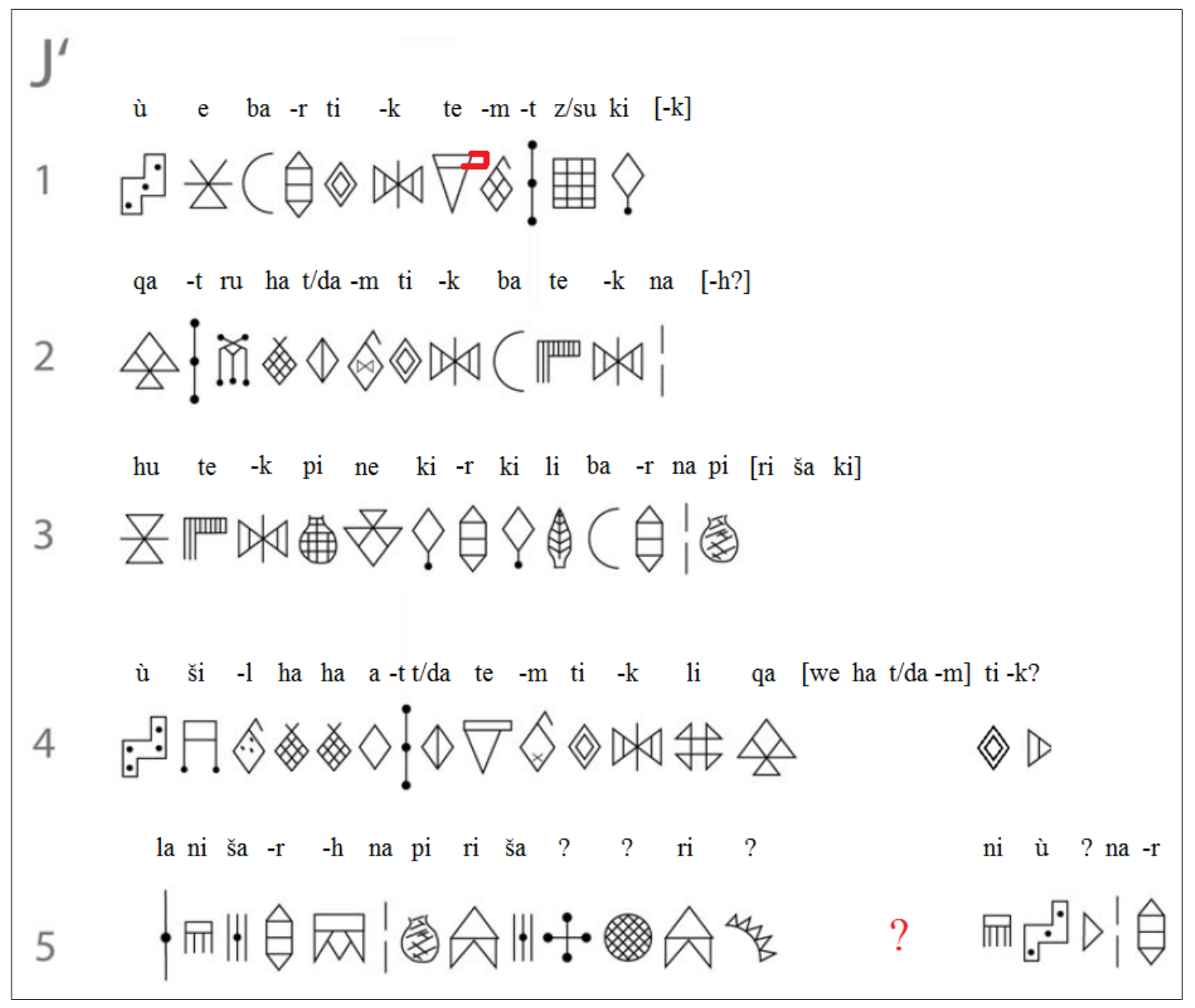


$\mathrm{K}^{\prime}$

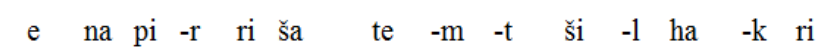

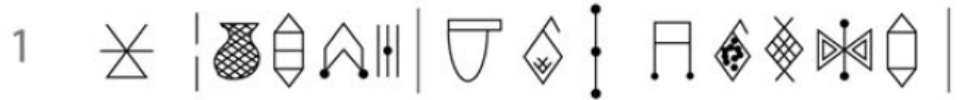

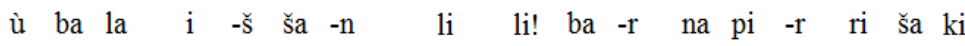

々C:

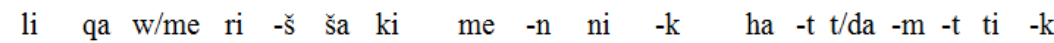

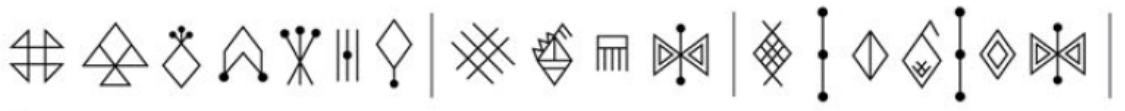

la -n ni ša ri $-\mathrm{h}$ ha ? ? ? ši ? ? na pi ri ša

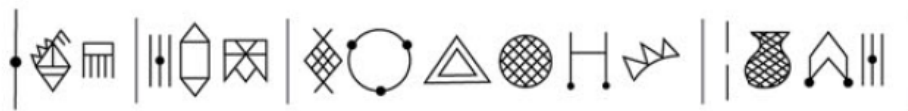

? ? ri ? t/da-h ? ? te - -n ti $\quad$ ?

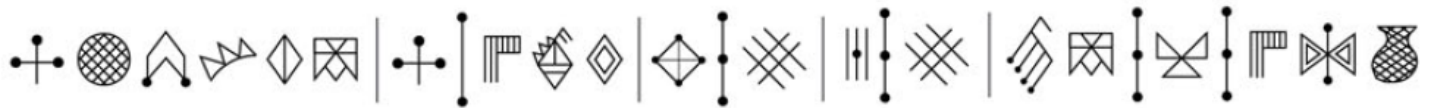

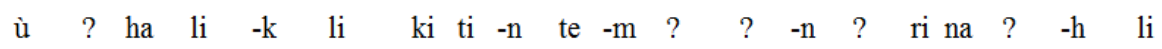

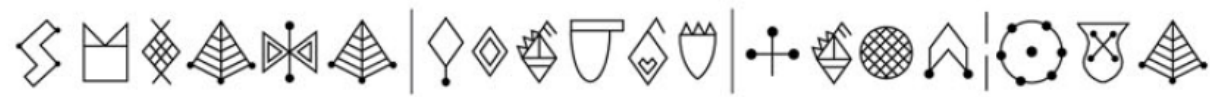

\section{Correspondances :}

La séquence suivante dans $\mathrm{Z}$ ligne 5 et $\mathrm{K}^{\prime}$ :

correspond à $n u$ ú te-en-ti dans [EKI 54] (nombreuses occurrences), [EKI 55 §9], [EKI 46 §18], [EKI 48 §21,32], [EKI 48a §5], [EKI 48b §5], [EKI $51 \mathrm{I}]$, [EKI 53 §] (voir aussi nu-um ú te-en-ti dans [EKI $47 \S \S 14,31,23]$ ).

Les séquences suivantes :

? -t me ša -t me a ? t/da $-\mathrm{k}$ me $\mathrm{t} / \mathrm{da}-\mathrm{k}$ me te ? ? ? ri na ? - $\mathrm{h}$ na

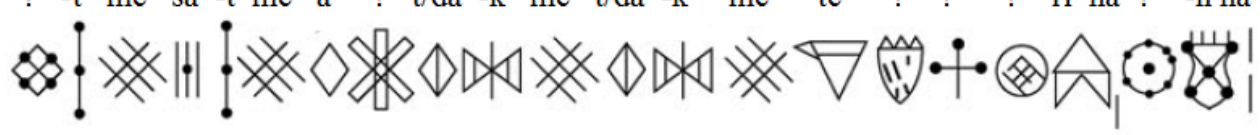

Y ligne 3 : 
Z ligne 9:

? me ša $-\mathrm{t}$ me $\mathrm{t} / \mathrm{da}-\mathrm{k}$ me te ?

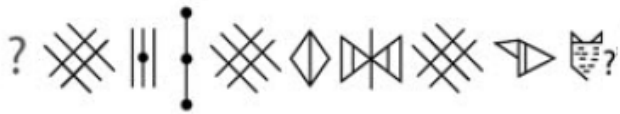

I' ligne 3 :

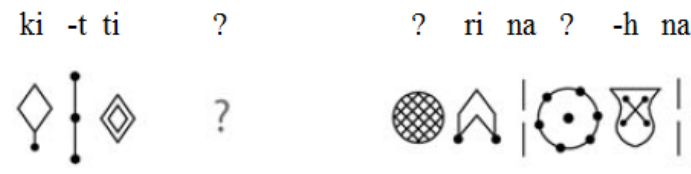

$\mathrm{K}^{\prime}$ (fin du texte) :

? -t me ša $-\mathrm{t}$ me ? $\quad$-h $-\mathrm{t} \quad$ ?

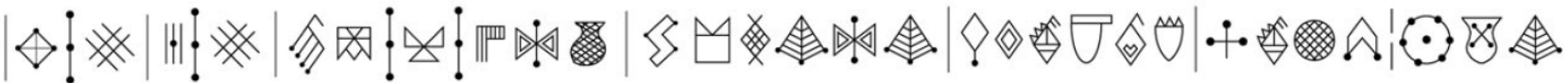

correspondent en grande partie aux formules (voir [EKI p.46 note 12] pour une vue synthétique) :

šu-út-me ša-at-me ki-it-ti-in me-lu-uk-ma te-im-ma gi-ri-na nu-un hi-h-na [EKI $13 \mathrm{VI}$ ]

šu-út-me ša-at-me ki-it-ti-in te-e-me hu-úr-du... [n]u-un gi-ri-na hi-ih-[li] [EKI $3 \mathrm{~V}, \mathrm{VI}]$

$\check{s} u$-ut-ti-me ša-at-ti-me ki-it-ti-in te-e-mi... [hu-ur-tu $\ldots$...nu-un gi-ri-na hi-h-hu-na] [EKI 48 §5] et

[EKI $53 \mathrm{I}]$

... gi-ri-na hi-h-li/ni [EKI 9 III a-c]

Les séquences suivantes :

Z ligne 5 :
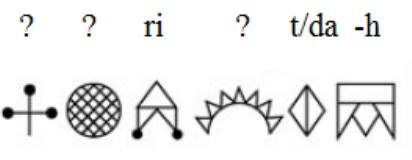

? ? ri ? ta $-\check{s}$

H'ligne $4: \bullet \cdot \widehat{8}$

? ? ri ? t/da $-\mathrm{h}$

F' ligne 2 :

? ? ri ?

I' ligne $2: \bullet \bullet 8^{\infty}$ ?

J'ligne $5: \stackrel{\bullet}{\bullet}$

? ? ri ? $\mathrm{t} / \mathrm{da}-\mathrm{h}$

$\mathrm{K}^{\prime}$ :

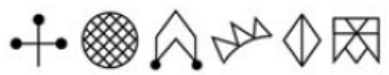


correspondent à gi-ri-ma ta-ah dans [EKI 54 §4]. De plus le verbe ta- ou tatta- est souvent précédé d'un mot portant le suffixe -ma.

De tout cela on peut déduire :

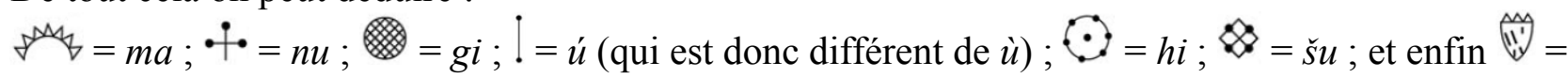
$m a, m e$ ou $m i$. Pour ce dernier signe, vu que d'autres signes correspondent de façon sûre à $m e$ et $m a$, la valeur $m i$ semble plus probable.

Les séquences suivantes dans $\mathrm{Y}$ et dans $\mathrm{Z}$ sont identiques et on peut reconstituer le texte entier en considérant les deux :

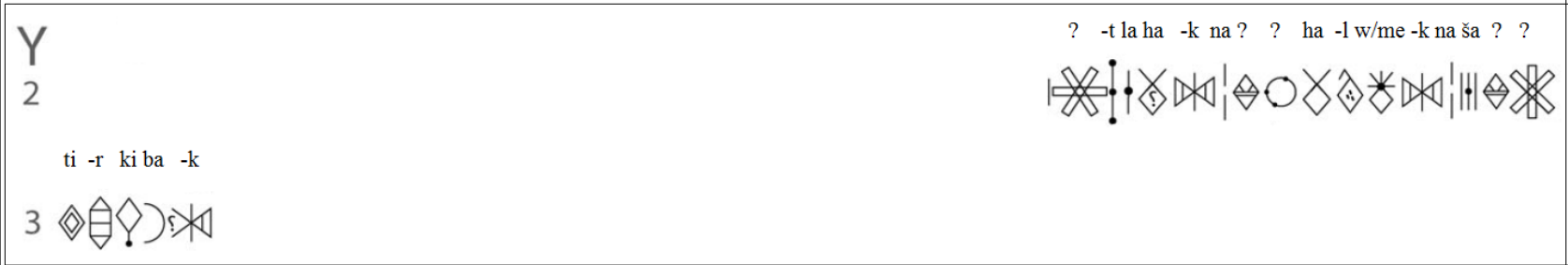

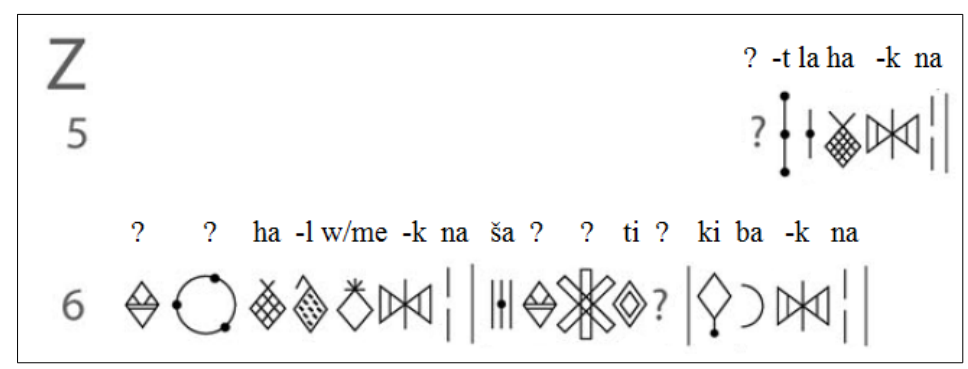

La séquence reconstituée se lit donc : lahakna $\hat{\nabla}$ halmakna ša $\hat{\nabla}$ tir kipakna

On trouve une correspondance partielle dans [EKI 3 VIII] :

$\left[?^{14}\right]$-it la-ha-ak-na [ti?]-a-te ha-al-ma-ak-na

Il est possible que le mot précédant lahakna (« qu'il disparaisse ») soit identique dans Y, Z et [EKI

3]. Pour le mot précédant halmakna (« qu'il soit banni ?»), cela semble moins probable.

On voit ici que $\diamond$ correspond à une lecture $m a$ en milieu de mot. La correspondance avec we/me est peut-être due à une réduction vocalique en fin de mot dans likaw/me.

14 Je n'ai trouvé nulle part de reproduction de l'inscription [EKI 3], ni de parallèle avec un autre texte, qui permette de supposer, au début de la ligne 28, un [hi] comme le fait l'entrée la-ha-ak-na de [EW:807]. Voir [EKI P1.2] et [MDP $31: 164]$ pour la reproduction du fragment $\mathrm{B}$ ou figure cette ligne. 
La séquence suivante dans $Z$ :

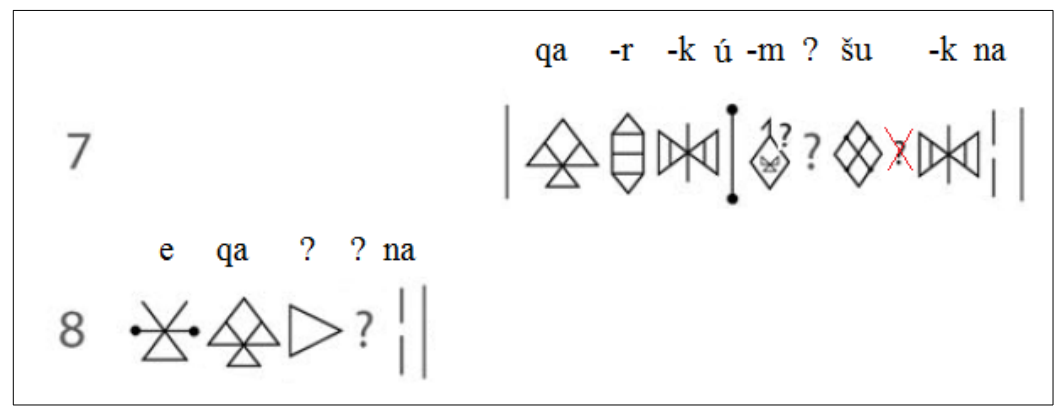

Ainsi que la séquence suivante dans Y :

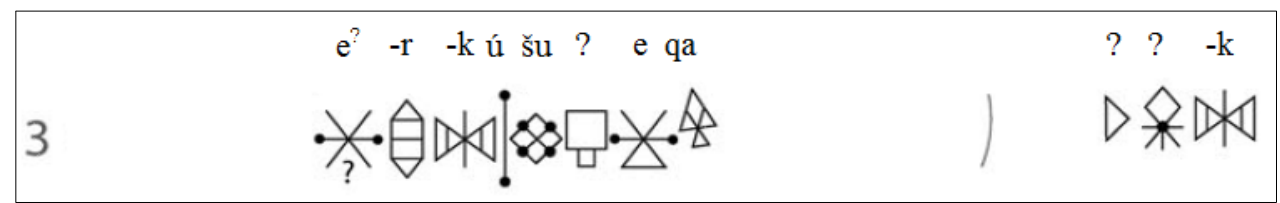

sont identiques à quelques variations près. On peut les rapprocher de [EKI 54 §73] « [...-a]r-ki inqa-az-za-ak-na », ainsi que du mot kark attesté dans [EKI 28A §2] qa-ar-ki-e, [EKI 28B I] qa-ar[ki-ú-me ...] et [EKI $46 \S 20] q a-a r-k i-u ́-[m e]$. Il semble bien que dans Y le mot soit $e-r-k$. La variation entre $\mathrm{Z}$ kark et $\mathrm{Y}$ erk est peut-être à mettre en parallèle avec celle entre $\mathrm{Z}$ šukna et $\mathrm{Y}$ šu-?

Dans Y, à la fin de la séquence, il est possible qu'un caractère । na ait été effacé. En effet l'inscription est endommagée à cet endroit, et l'espace apparemment vide fait à peu près la largeur d'un caractère :

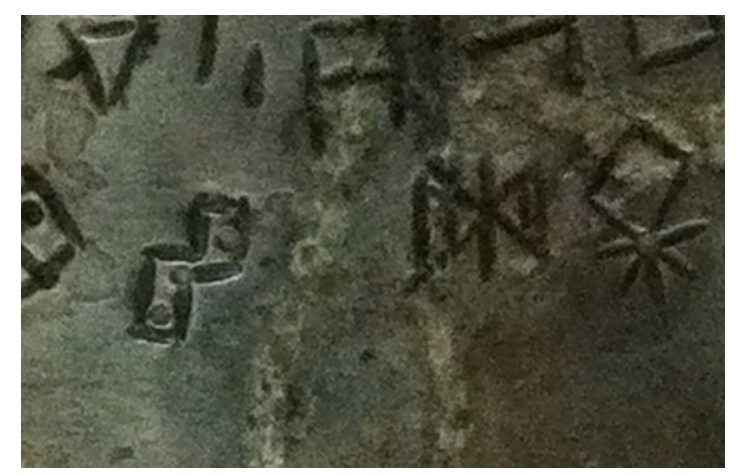

Figure 5 : partie endommagée de Y, ligne 3.

Un caractère peut avoir été effacé (au centre)

On peut donc déduire $\checkmark=-z$ et $\stackrel{\hat{*}}{=}=z a$. Cette dernière lecture est confirmée par le fait que les deux inscriptions associées à des représentations féminines, I et $\mathrm{Q}$, et seulement celles-ci, commencent par le mot zana « dame ». Cela avait déjà été proposé par Meriggi ([Meriggi 1971:196]). 
La séquence suivante dans Z :

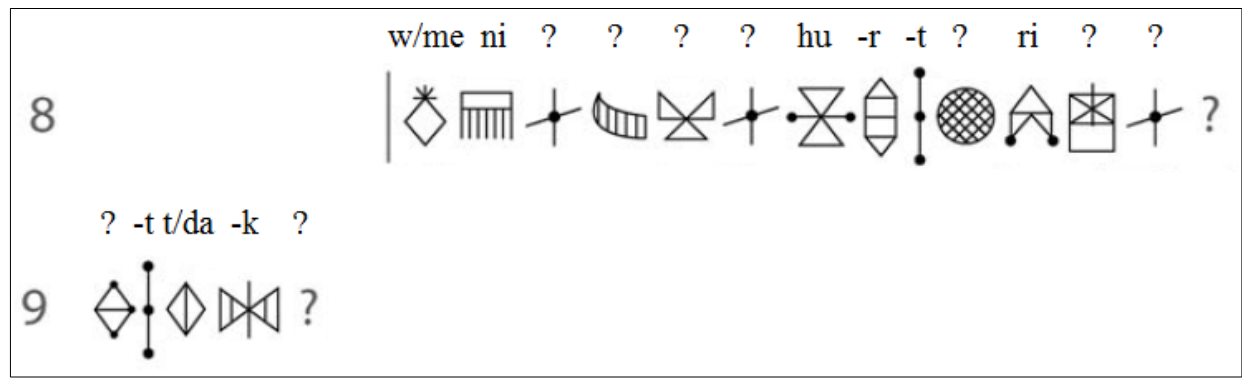

[EKI 3 VII] ma-ni-ip sa-hi-[i] ${ }^{15}$ hu-úr-du gi-ri-we [ku-up ?] ${ }^{16}$ hu-[út]-ta-ak-na

[EKI 54 §14] hu-ub-ba sa-hi-i gi-ri-pa ku-up hu-ut-ta-[a]k-na

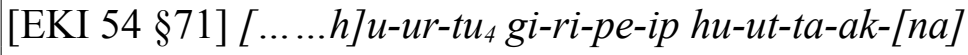

[EKI 45 §8] ma-ni hu-ur-tu4 gi-ri-pu-up hu-ut-tak-n[a]

On peut proposer deux déchiffrements pour la séquence dans $Z$ :

ma-ni-p sa-hi-p hu-r-t gi-ri-w/pV-p hu-t-ta-k-[na]

ma-ni-p ? ku-p hu-r-t gi-ri-w/pV-p hu-t-ta-k-[na]

On trouve ici $\searrow=m a$ en début de mot.

On peut déduire $=w / p V$, où $V$ est une voyelle mal définie entre $e, a$ et $u$.

On déduit aussi $\nabla=h u$ et ${ }^{-}-=-p$.

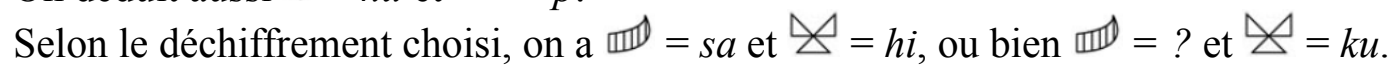

Plus hypothétiquement, la séquence suivante de Z :

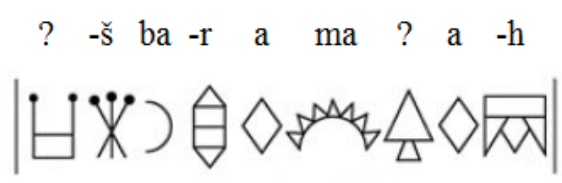

Dont le début pourrait correspondre à [EKI 45 §20] ku-uš ba-ri-e « sa descendance ».

On aurait donc $\forall=k u$ ?

Dans Z, la séquence $z /$ sun $z /$ sun dans le passage suivant :

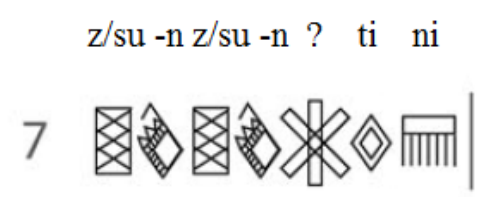

est peut-être à rapprocher de šú šú dans [EKI 38 X] et šú dans [EKI 37 II], qui est interprété comme une particule de confirmation comparable à « amen » (voir [EW:1173]).

15 Reconstitué par König d'après [EKI 54 §14]. On pourrait aussi proposer sa-hi-[ip] d'après Z.

16 Reconstitué par König d'après [EKI 54 §14]. On pourrait aussi proposer gi-ri-we-[ip] hu-[út]-ta-ak-na d'après $Z$, [EKI 54 §71] et [EKI $45 \S 8]$. 


\section{Authenticité de L'}

Comme on peut le voir dans les images ci-dessous, l'inscription L' est constituée de morceaux de Y recopiés et collés ensemble de façon plus ou moins aléatoire. On note une confusion entre $! \dot{u}, \vdots:-t$ et la simple barre verticale utilisée comme séparateur. En effet à trois reprises, la séquence copiée commence ou se finit par ! ou : résultant en une coupure au milieu de mots :

- [qa]-t-ru recopié sans restituer le [qa], qui est effacé dans Y, et tronqué au niveau de : - $t$;

- $\check{s} u$-? e-qa-z-za-k-na est recopié en tronquant le mot au milieu au niveau du $\checkmark-z$, et sans comprendre que le $\Delta$ $q a$ fait partie de la ligne 3 et pas de la 2 ;

- $\quad i l i-k a-w / m e ~ i-t a-t-t u-r i$ est tronqué au niveau de $:-t$, et écrit dans le mauvais sens. En effet, cette partie du texte est à lire de gauche à droite dans $\mathrm{Y}$, contrairement au reste du texte.

Enfin, le caractère $\Downarrow_{t a}$ encadré en rose est partiellement effacé dans Y, et recopié tel quel dans L'.

Toutes ces caractéristiques dénotent une méconnaissance totale du système d'écriture de l'élamite linéaire, et montrent que L' n'a pas été rédigée par un scribe élamite. L'inscription est donc très probablement un faux, peut-être ajoutée sur un objet véritablement élamite pour en augmenter la valeur marchande. La maison de vente aux enchères qui a mis l'objet en vente semble ne pas avoir été dupe, exprimant ses doutes dans la description de l'objet (c'est moi qui souligne) : " A sheet silver vessel with domed plaque to the base, waisted profile, everted rim, band of ProtoElamite text beneath the rim, text possibly a later addition ${ }^{17}$
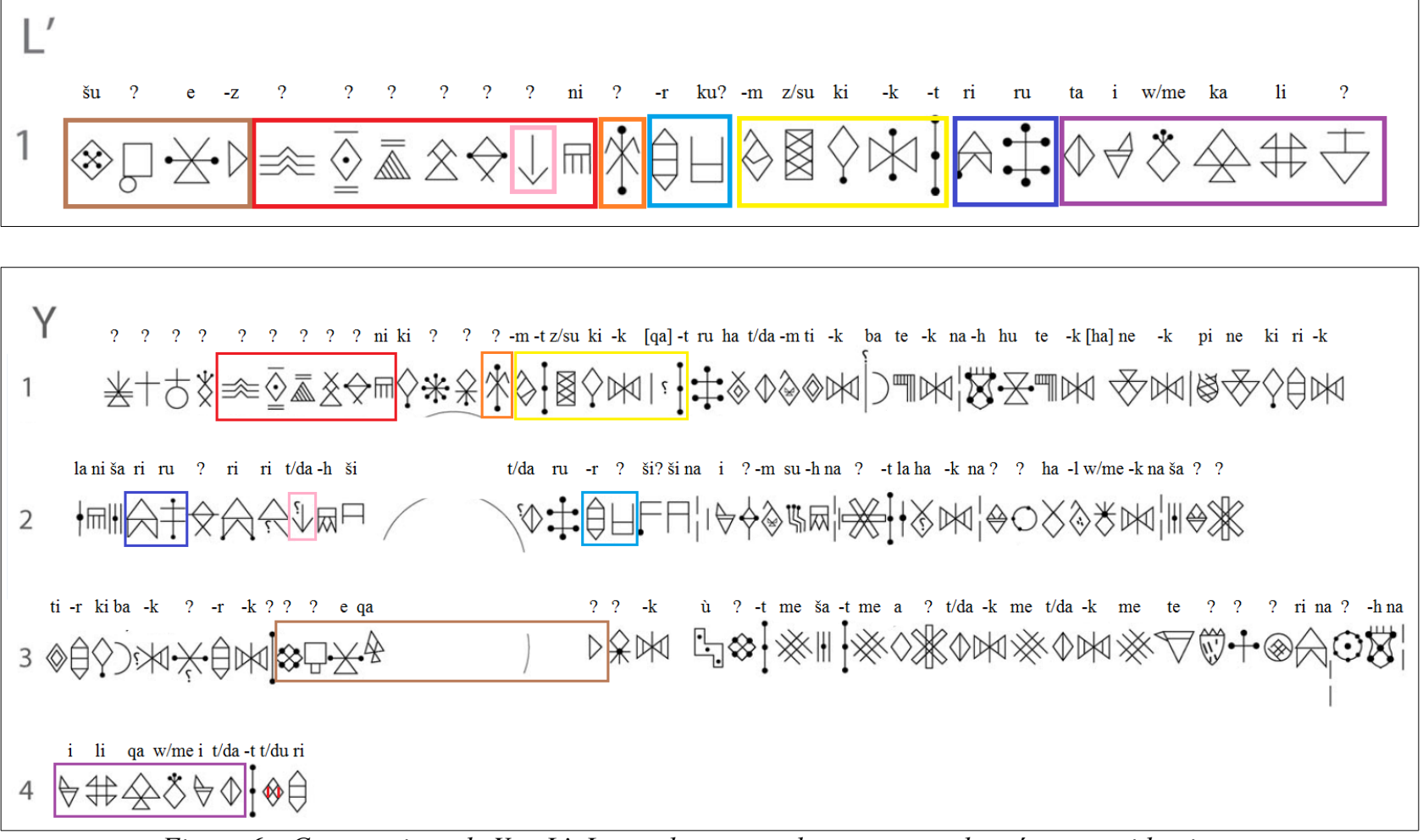

Figure 6 : Comparaison de Y et L'. Les cadres en couleur montrent les séquences identiques.

17 Source : https://web.archive.org/web/20160118165551/https://www.timelineauctions.com/lot/silver-vessel-withproto-elamite-text/60060/ 


\section{Résultats intermédiaires (troisième étape)}

\begin{tabular}{|c|c|c|c|c|}
\hline 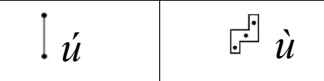 & $\theta_{i}$ & $\forall_{e}$ & $\nabla_{a}$ & \\
\hline DO pu & \multicolumn{2}{|c|}{$p i$} & $C_{b a}$ & $+-p$ \\
\hline \multicolumn{4}{|c|}{$\otimes=w / p V(V=e / a / u)$} & \\
\hline$\otimes t u / d u$ & $\Delta t i$ & $\nabla t e^{18}, \| m b e$ & $\checkmark t a / d a$ & $\vdots-t$ \\
\hline$\forall=k u^{?}, \triangle k u^{? 19}$ & \multicolumn{2}{|c|}{$\hat{\vartheta}_{k i}, g i$} & $\Delta_{q a}$ & 战 $-k$ \\
\hline\langle\rangle$_{s u / s ̌}, \check{s}_{\check{s} u}$ & \multicolumn{2}{|c|}{ 月 ${ }_{s i}$} & $|\oplus|_{\breve{s} a}$ & $\Psi_{-\check{s}}$ \\
\hline \multirow[t]{3}{*}{$\theta_{z u / s u}$} & & & 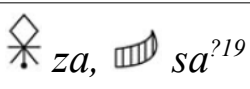 & $\triangleleft_{-z}$ \\
\hline & 清 $\mathrm{mi} / \mathrm{ma} / \mathrm{me}$ & 州me & $+4 m a$ & $\hat{\otimes}-m$ \\
\hline & & \multicolumn{2}{|c|}{$\bigotimes_{m a / w e / m e}$} & \\
\hline \multirow[t]{2}{*}{$\bullet \cdot n u$} & mint $20 n i$ & $\nabla_{n i(/ n e /)}$ & $n a$ & 部 $-n$ \\
\hline & \multicolumn{2}{|c|}{ 壮 $l i$, $l i$} & †la & 金 $-l$ \\
\hline$\because{ }^{21} r u$ & \multicolumn{2}{|c|}{$\hat{A} r i, \hat{\theta} r i$} & & $\theta-r$ \\
\hline$\nabla h u, \nabla h u$ & \multicolumn{2}{|c|}{$\therefore h i, \triangle h i^{? 19}$} & $h a$ & 肉 $-h, \otimes-h$ \\
\hline
\end{tabular}

Figure 7 : équivalences entre élamite linéaire et valeurs de l'élamite cunéiforme

18 La syllabe notée par $\nabla$ est probablement distincte de celle notée par $\|^{\| m}$.

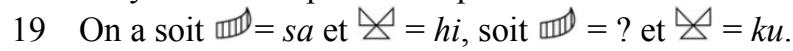

20 Variante graphique: $\exists$

21 Variante graphique (ou phonétique) : $\mathrm{m}$ 


\section{Déchiffrement de Q}

Les valeurs trouvées nous permettent de déchiffrer le texte Q en grande partie.

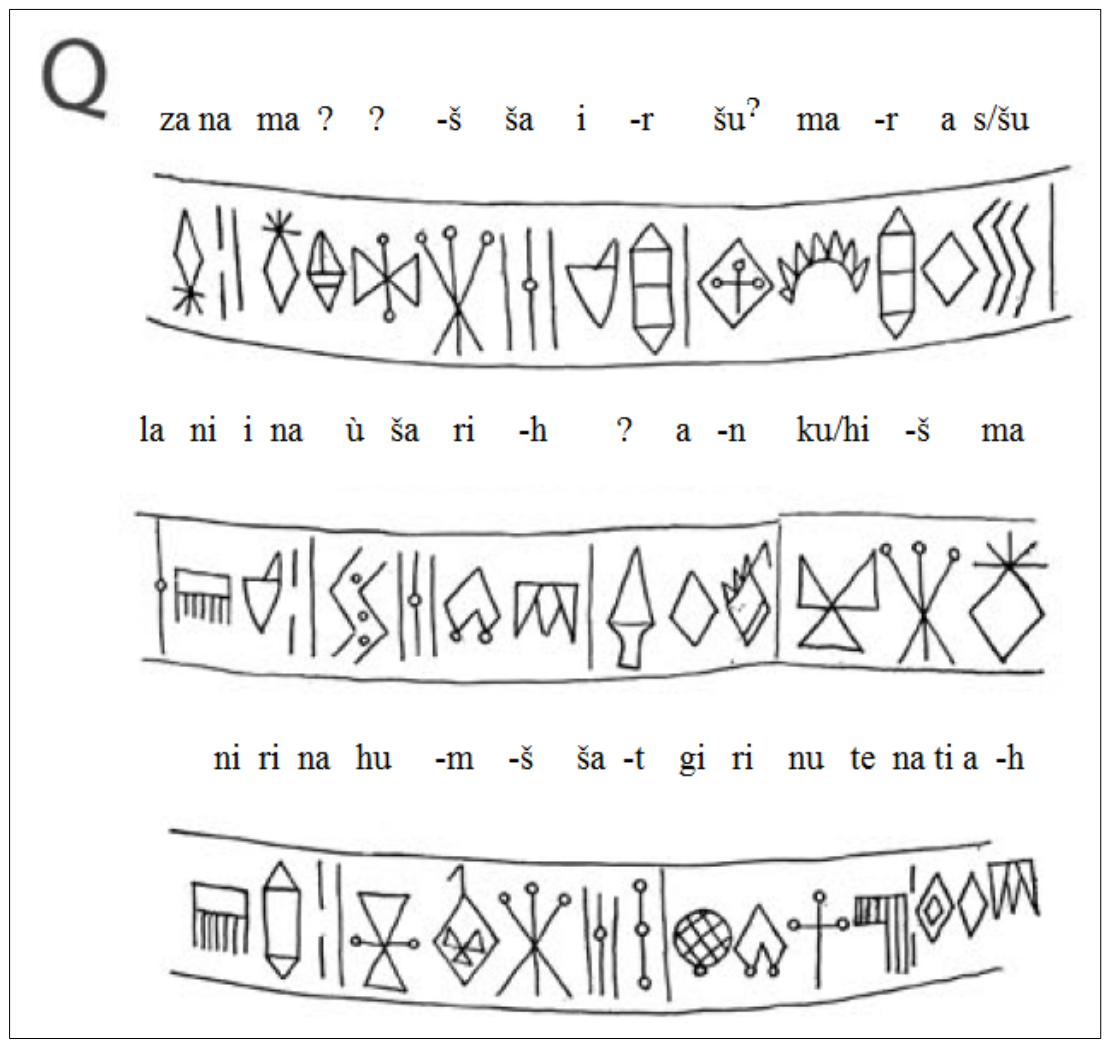

On note un long parallèle entre Q et A', qui commence par laniina ù šarih et se termine par Humššat giri nutena tiah dans Q et Humban giri nutema tiah dans A'.

On en déduit que Humššat est un nom de divinité (peut-être le correspondant féminin de Humban). Ce nom se retrouve peut-être dans le traité de Naram-Sin ([MDP 11:1-11]). En effet, dans ce dernier on trouve (recto, Col. 1 ligne 23) le nom de divinité ${ }^{d} H u-u m-q a^{3}-a t^{\prime}$. La lecture qa est donnée comme douteuse par Scheil car la partie gauche et basse du caractère est effacée. Le nom est lu huum-qa-at sans hésitation dans [EKI 2 §1].

Vu la disposition de la partie effacée et la ressemblance de la partie droite des caractères $q a$ et $\check{s} a$, il est peut-être envisageable de reconstituer $u m-\check{s} a$ au lieu de $u m$-qa qui est la lecture retenue jusqu'à présent. 


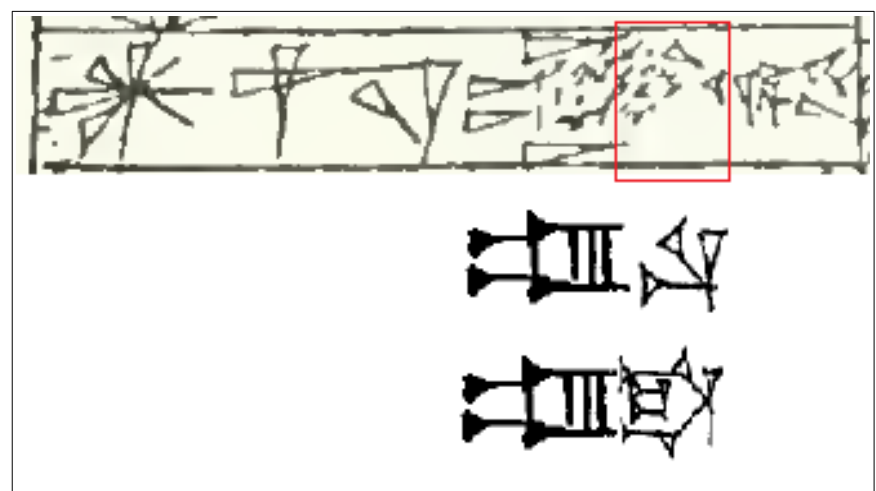

Figure 8 : En haut, ${ }^{d} H u$-um- $q a^{2}-a t^{2}$ Dans le traité de Naram-Sin ([MDP 11:3]). Le rectangle rouge correspond au signe $q a^{?}$ dans l'autographie de Scheil.

Au milieu : reconstitution um-qa; en bas : reconstitution um-ša.

Le caractère $\&$ qui figure au début de $\hat{\imath}-a-n$ dans Q pourrait être le même que celui qui figure dans Z, ligne $3: k u^{?}$ šbar ama $\triangle-a-h$.

Si on pose $\hat{\mathrm{S}}=\widehat{\Delta}=s i$, on trouve le mot si(y)an « temple » dans $\mathrm{Q}$, et si(y)ah « j' ai regardé » dans $\mathrm{Z}$ (voir [EW:1096]).

Dans Z, la phrase entière pourrait signifier « j'ai regardé la descendance de (ma?) mère »; cela fait d'autant plus sens que la phrase précédente se termine par hahpuh «j'ai écouté ».

Ce texte pèse plutôt en faveur de la lecture $\triangle=k u$ (et donc uill ne devrait pas être lu $s a$, et reste inconnu).

- kušmanirina peut être mis en parallèle avec ku-uš-ma-ni ([EW:568]) « est à construire » et se rapporterait au temple : « un temple devant être construit pour Humššat » ou « Humššat dont le temple doit être construit »;

- je n'ai trouvé aucune interprétation possible pour hišmanirina (ni pour manirina si on détache hiš « nom »). 


\section{Textes de Suse : les textes A, B, C, et E}

Pour ces textes nous adoptons la proposition $\|=z u$ (en plus de $D O=p u$ précédemment), ce qui permet de lire le nom de Puzur-Šušinak. De plus, il est possible que $\|$ soit une variante de 图 utilisée sous son règne.

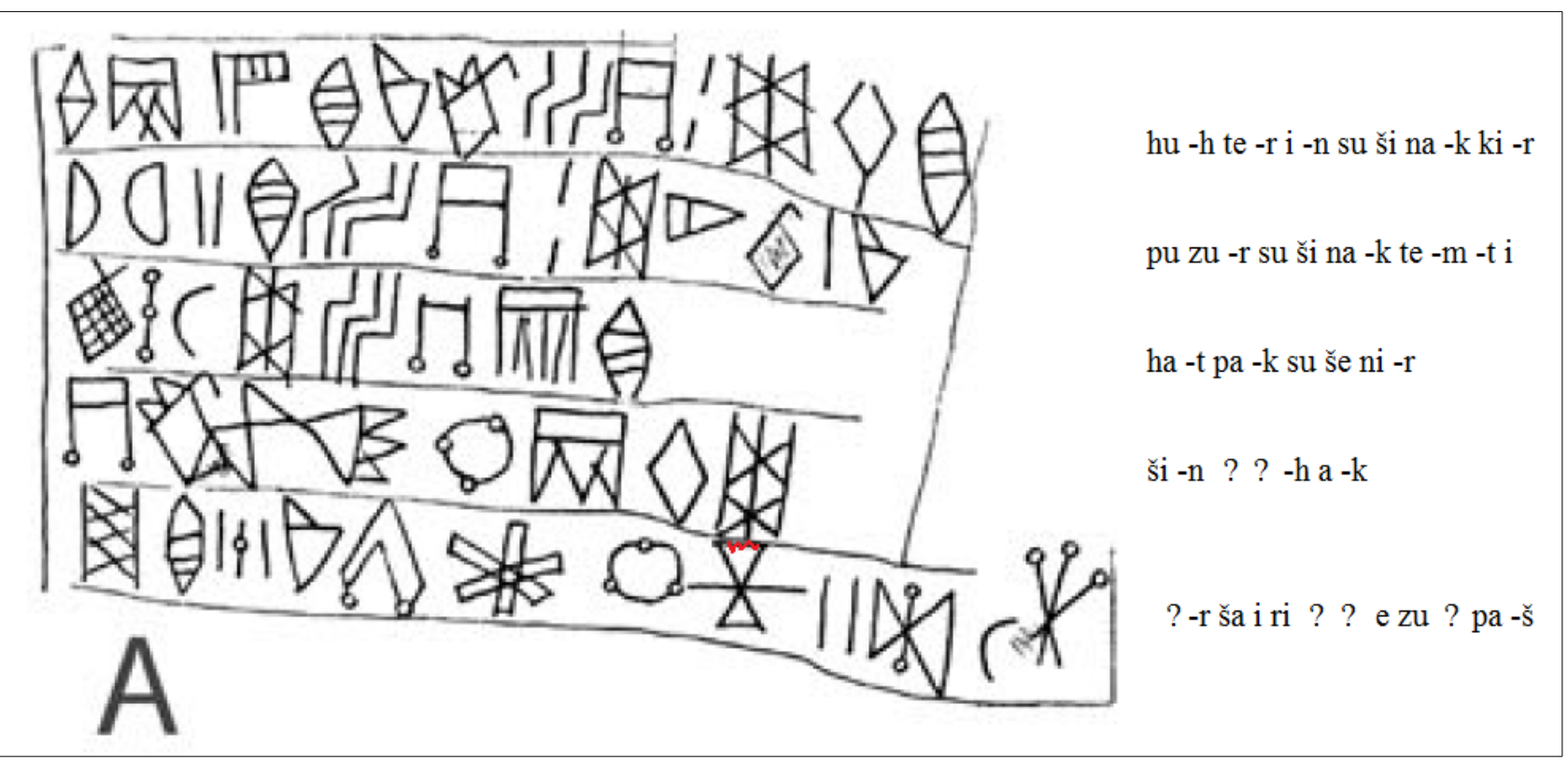

On peut reprendre ici la proposition $\$=p i$ de [Mäder et al. 2017:35], déjà évoquée dans la note 12 . On peut mettre en parallèle ce texte avec la première colonne du texte akkadien (voir [Gelb \& Kienast 1990:328-329] et [Sollberger \& Kupper 1971:124-125]), et lire les trois premières lignes de la façon suivante :

huh ter Insušinakkir

Puzur-Sušinak temt $i$ hatpak Sušenir

Šinpišuh ak
Destiné au bienveillant Insušinak

Puzur-Sušinak à son seigneur (ou : P.-S. le seigneur ?)

ensi de Suse

fils de Šinpišuh

Avec les interprétations ci-dessous, le texte élamite peut être considéré comme l'équivalent du texte akkadien, à l'exception du titre « šakkanakku du pays d'Elam » qui manque en élamite.

huh ter Insušinakkir : l'équivalent dans le texte akkadien est « en l'honneur d'Insušinak ».

- Pour $h u h$, voir $h u-h$ [EW:684]. L'interprétation $h u h$ « destiné à » convient dans toutes les occurrences données et fait plus sens que la proposition « plein(?)» de Hinz \& Koch :

- ud-du.KI.MIN hu-h v.ha-tan-tuk-iš « le sacrifice nécessaire destiné à l'oracle »

- hu-h si-a-an $<$ d. $>$ DIL.BAD « destiné au temple de $<$ la déesse $>$ (astre) Vénus »

- h.A.lg [hu(?)]-h h.GIŠ.MÁ.lg.na « le fleuve était destiné aux navires (navigable)»

- $\quad$ Pour ter « favorable, bienveillant», voir [Grillot-Susini 2008:19] $]^{22}$.

22 Il est possible qu'on retrouve le même mot dans les expressions nu-te Napiriša (X), nu-te-ma Napiriša (I'),

Humban giri nu-te-ma tiah (A'), Humššat giri nu-te-na tiah (Q). 
Puzur-Sušinak temt $i$ : la syntaxe est étrange. Logiquement temt $i$ doit être l'équivalent de bēlisu " son seigneur ", on s'attendrait donc à quelque chose comme : huh ter Insušinak temt i PuzurSušinak etc. A moins que temt ne désigne Puzur-Sušinak lui-même, et que $i$ soit utilisé comme démonstratif?

hatpak Sušenir : hatpak est l'équivalent de ensi. On peut le décomposer en deux éléments :

- hat, cf ha-at « bâton (de commandement), sceptre (emprunté à l'akkadien hatṭu)» dans [EW:581-582].

- $\quad p a k$ « qui détient », cf ba-ak-ra de même sens en néo-élamite dans [EW:106].

Šinpišuh ak : il faut admettre que c'est l'équivalent de mâr Šimbi-išhuk « fils de Šimbi-išhuk », et que $a k$ soit un mot signifiant « fils », ou un suffixe de sens équivalent. Une fois de plus la syntaxe est étrange : pas de suffixe de classe (on a bien Šinpišu akir dans G et H, mais pas ici), et dans les textes plus tardifs on rencontre plutôt la formule $\check{s} a k X$.

Une forme proche de Šinpišuh (ou Šinpišu dans G et H) est attestée dans une tablette comptable, akkadisée en Šim-bi-iš-hu ([Steinkeller 2013:299]).

On peut supposer que la dernière ligne correspond aux deux dernières lignes de la première colonne du texte akkadien " a voué un clou (de fondation) en cuivre et en cèdre ». Il est possible de faire correspondre le texte élamite à cette traduction avec la lecture suivante :

pur ša iri ha? šsue $e^{23} z u p ? p a s ̌ \quad$ un clou de fondation en cuivre [et] en cèdre il a voué

pur : équivalent de l'akkadien sikkat « clou ». Voir [MDP 53:20] avec le sens « fondation ».pu-ur dans [EW:241] doit être interprété dans ce sens.

$\check{s} a$ : voir l'entrée dans [EW:1109] avec le sens possible de « de (provenance) ».

iri : voir e-ri-ni dans [EW:401], emprunté à l'akkadien erû « cuivre », qui est précisément le mot équivalent dans la version akkadienne du texte. On peut éventuellement lire $i r i<n>$, avec un $-n$ final omis, comme souvent en élamite linéaire.

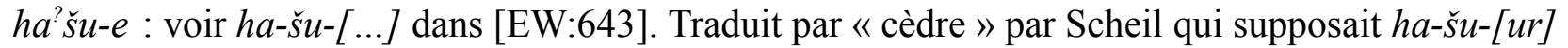
dans l'hypothèse d'un emprunt à l'akkadien hašûru " un genre de cyprès ». L'équivalent dans le texte akkadien est erini. Le - e final peut soit faire partie de la racine, soit être une forme du suffixe ia qui désigne la matière d'un objet (voir [Grillot-Susini 2008:49]).

zup? paš : l'identification en tant que forme verbale équivalente à l'akkadien išruk est évidente, de par le placement en fin de phrase et la terminaison verbale $3^{\text {ème }}$ pers. sg. Voir $z u-u b-b a-h$ dans [EW:1312], de sens douteux.

Si on admet ces interprétations assez hypothétiques, on aurait :

政 $=h a$ et $\bowtie=-p$. A ce stade, il vaut mieux les considérer comme de simples possibilités. 
Lecture « $e$ »du caractère 8 de la ligne 5 :

En regardant la photographie de A dans [Hinz et al. 1969, p.28], il semble qu'il ne faille pas

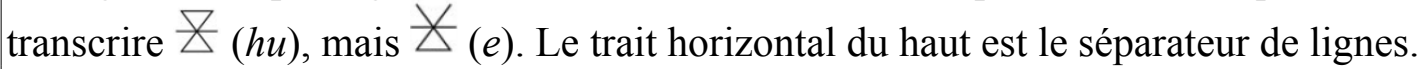

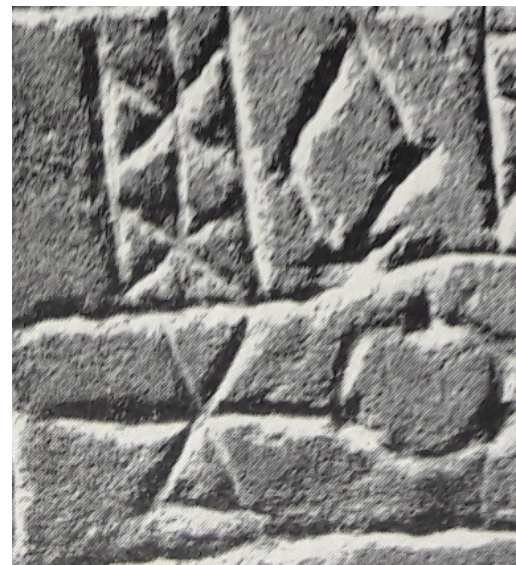

Figure 9 : détail de la photographie de A dans [Hinz 1969, p.28]

Les textes $\mathrm{C}$ et $\mathrm{E}$ se ressemblent fortement, si bien qu'on peut les utiliser pour se compléter l'un l'autre.

Ils contiennent le titre absent dans A, mais largement attesté dans la titulature de Puzur-Inšušinak « šakkanakku du pays d'Elam », écrit GİR.NÍTA māti NIM ${ }^{K I}$ en akkadien (voir [Alvarez-Mon 2019:172]). En élamite, ce titre prend la forme $\check{s} p^{?} k$ hurt hatamtipir.

On reconnait dans cette expression le mot hurt « peuple»; mātu peut aussi avoir ce sens en akkadien, voir [Assyrian Dict 10:414]. Le mot šep? $k$ doit être l'équivalent de šakkanakku ; on le retrouve dans A'. Le dernier mot n'est totalement lisible sur aucune inscription, cependant on peut déduire que c'est une forme de hatamti, ce qui est cohérent avec la forme en losange des caractères dans E. Il est probable qu'un caractère pi pluralisant soit présent, comme dans les expressions $h u$ $u$ r-du an-ša-ni-ip-na a-gi šu-še-ni-ip-na [EKI $3 \mathrm{VI}$ ] et hu-ur-ti ha-da-am-ti-ip-na [EKI 70C V] et hu-ur-tu ${ }_{4}$ an-ša-ni-ip-na a-ak šu-še-ni-ip-na [EKI 53 I]. Voir [EW:722-723] pour l'ensemble des occurrences de hu-úr-du, hu-ur-ti, hu-ur-tú et hu-ur-tu .

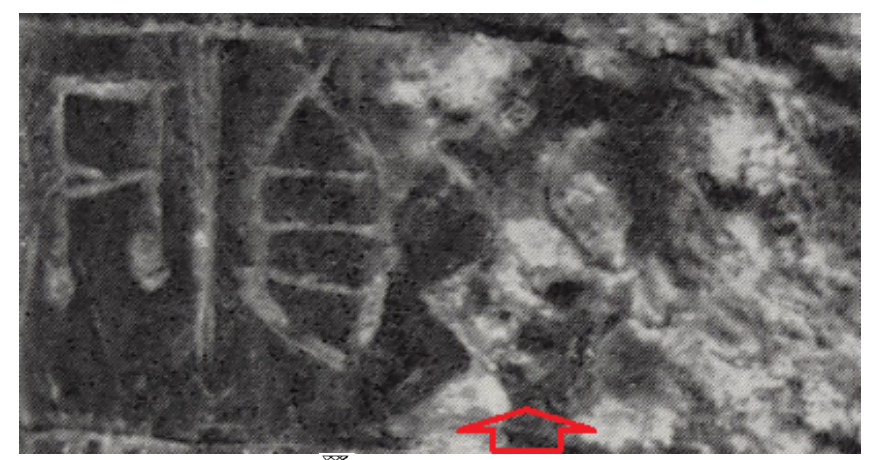

Figure 10 : trace du caractère pi à la ligne 3 de C (voir [Hinz 1969:31-33])

Le sens de šep? $k$ hurt hatamtipir est donc « šakkanakku du peuple des élamites ». 


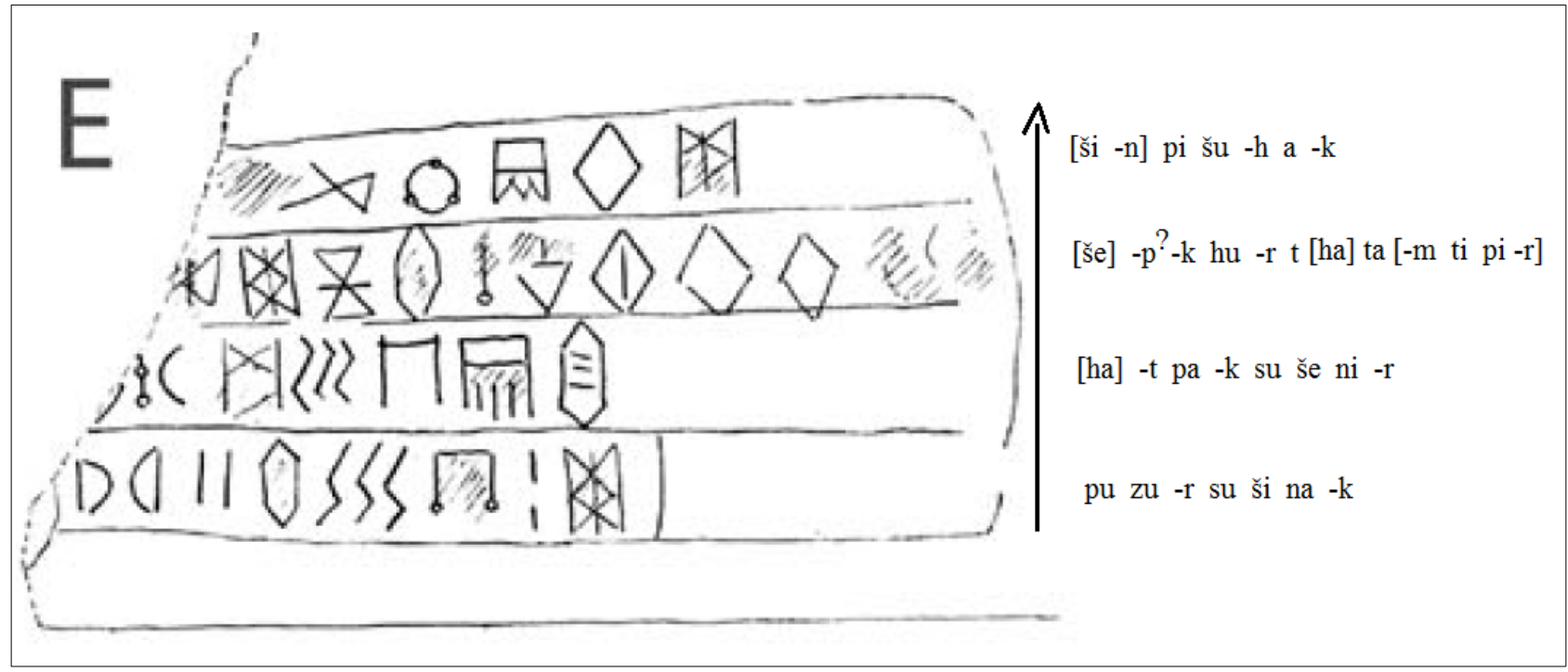

\section{Puzur-Sušinak}

[ha]tpak Sušenir

[še]p? k hurt [ha]ta[mtipir]

[Šin]pišuh ak

Dans $\mathrm{C}$, le texte se poursuit, peut-être à l'identique de A. En effet la zone abîmée sous la ligne 3 pourrait constituer une ligne 4 assez longue pour contenir les six caractères [šu e zu $\left.-p^{?} p a-\check{s}\right]$.

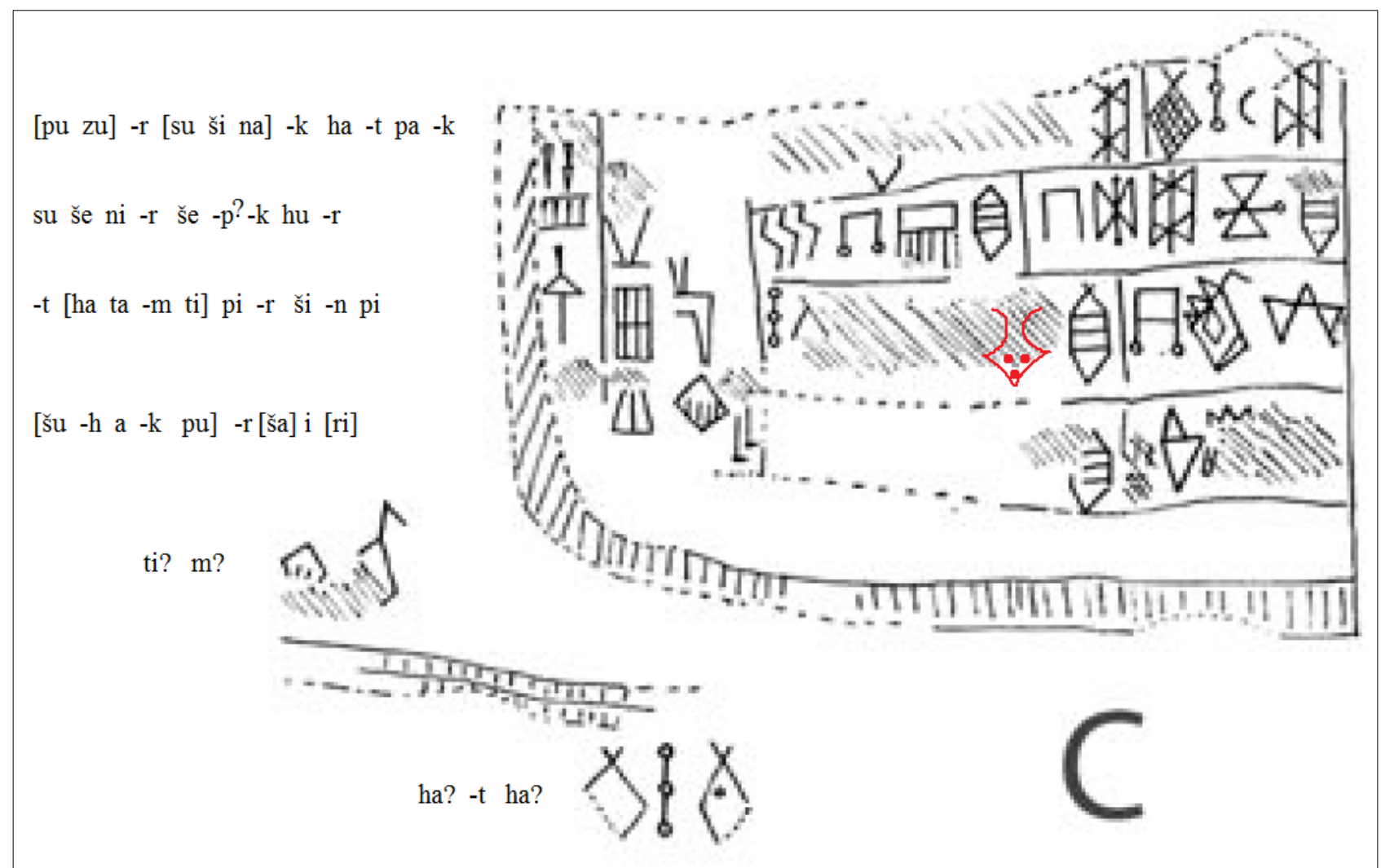

\section{[Puzu]r-[Sušina]k hatpak}

Sušenir šep?k hur-

-t [hatamti]pir Šinpi-

[-šuh ak pu]r [ša] i[ri ha ${ }^{3}$-]

[-šue zup? paš]? 


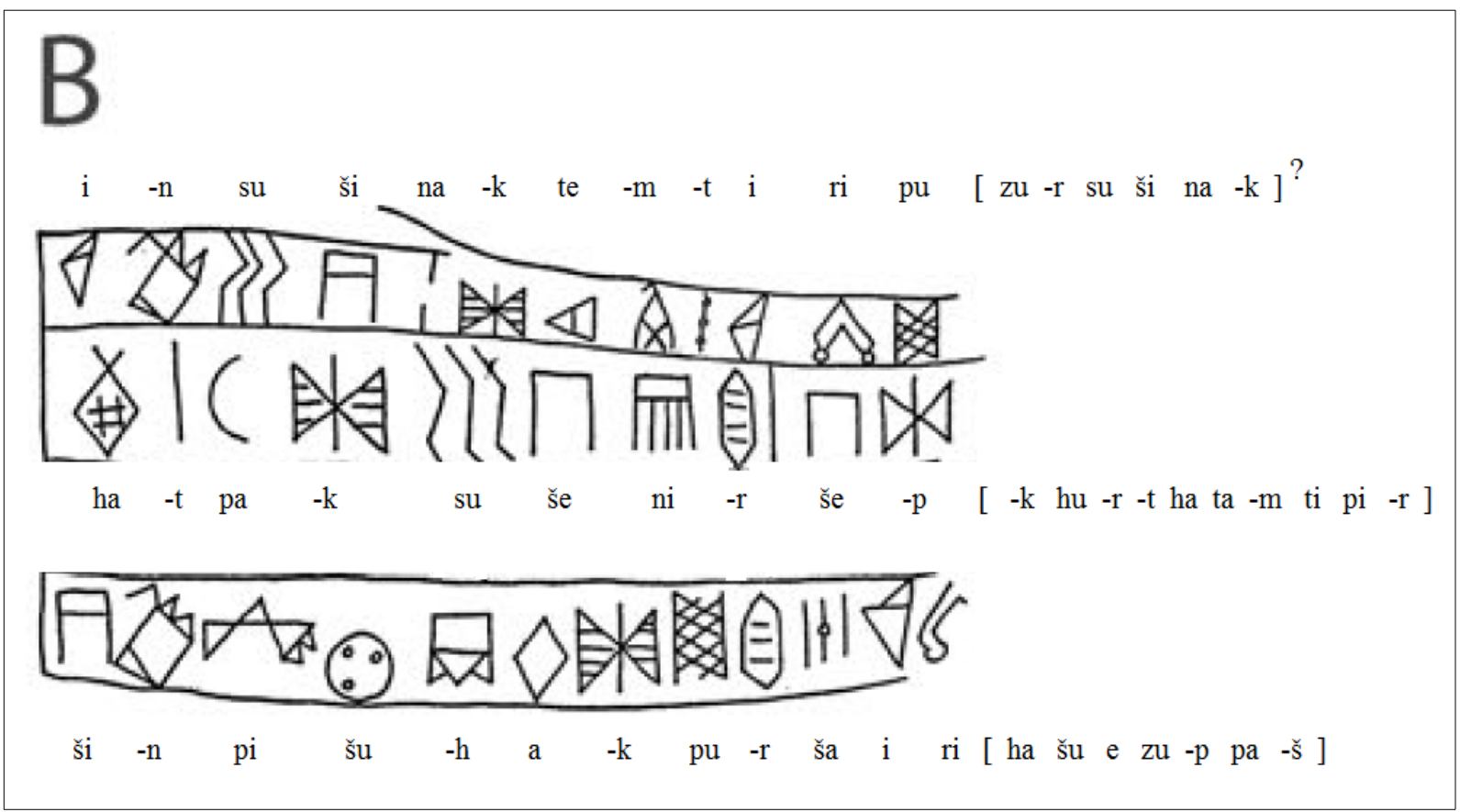

Le texte $\mathrm{B}$ ressemble lui aussi à $\mathrm{A}, \mathrm{C}$ et $\mathrm{E}$, avec des variantes :

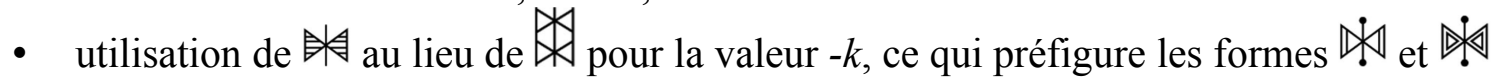
utilisées plus tard;

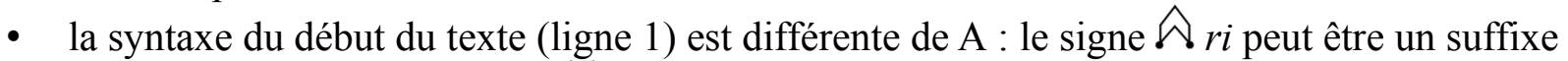
de $3^{\text {ème }}$ personne. Dans ce cas, ${ }^{2}$ pu pourrait être le début du nom de Puzur-Sušinak, dans une variante alternative à la plus courante, qui utilise $\mathrm{DO} p u$.

Insušinak temt-iri pu[?zur-Sušinak]

hatpak Sušenir šep? [k hurt hatamtipir]

Šinpišuh ak pur ša iri [ha? šue zup? paš] à Insušinak son seigneur, $\mathrm{Pu}[$ ?zur-Sušinak]

ensi de Suse, šakka[nakku du peuple des élamites,]

fils de Šinpišuh, un clou de fondation en cuivre [(et) en cèdre il a voué.] 


\section{Textes de Suse : les textes F, G et $\mathrm{H}$}

Pour ces textes, l'article de référence est [André \& Salvini 1989].

Pour F, la première moitié de la ligne du bas peut être restituée par comparaison avec $\mathrm{G}$ et $\mathrm{H}$, la deuxième moitié est moins sûre.

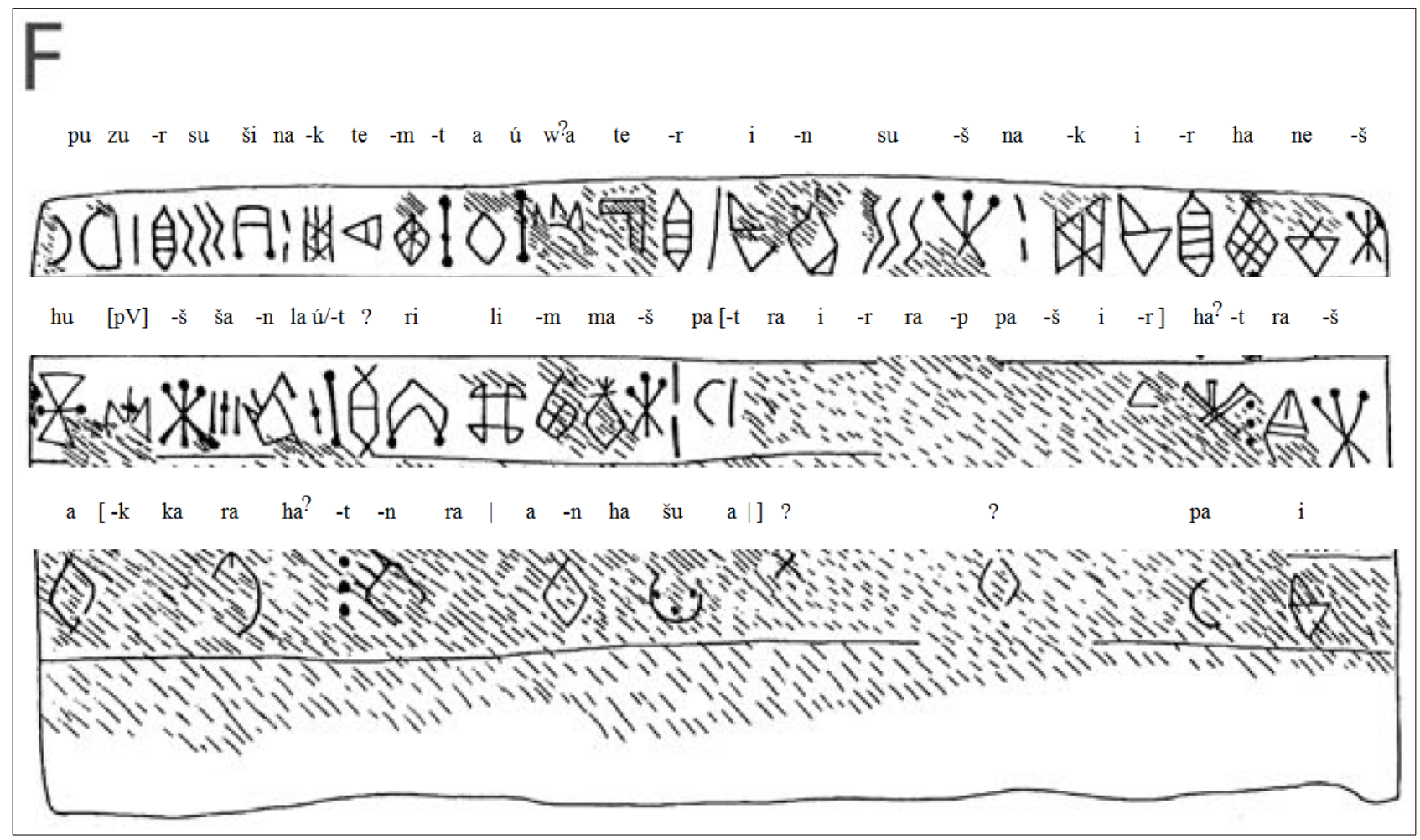

Puzur-Sušinak temt aúm/wa ter | Insušnak ir haneš

hu[pV]ššan la-(ú/t)-Øৈ-ri limmaš | pa[tra ir rappaš ir] ha? traš

a[kkara ha? tnra $\mid$ an-ha-šu-a ...? ... ]

Pour $\mathrm{G}$, on voit que la première moitié de chaque ligne doit manquer. On peut la restituer de façon convainquante, car le nombre de caractères manquants est le même sur chaque ligne : 15 (ou un peu plus) sur la première, 15 sur la deuxième, et 14 sur la troisième. Il avait déjà été noté dans [André \& Salvini 1989:62] que G « est un duplicata de la partie gauche finale de $\mathrm{H}$ ». 
G

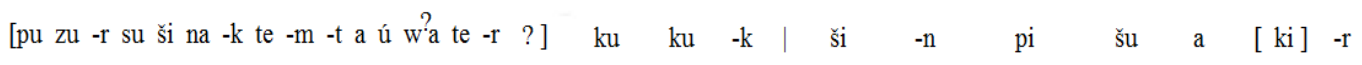

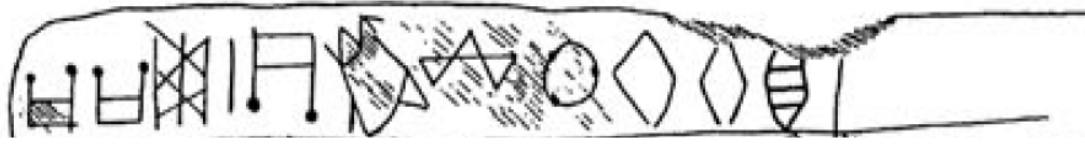

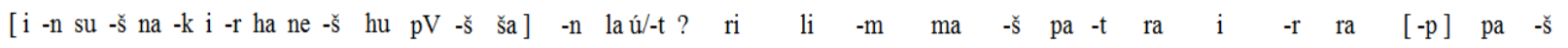

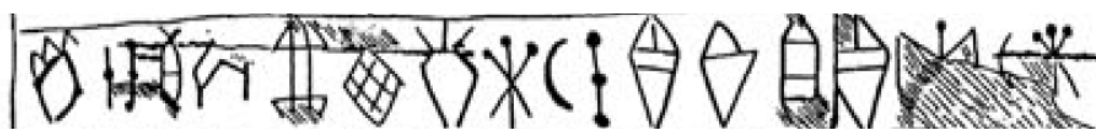

[ i -r ha ${ }^{?}-\mathrm{t}$ ra - š a $-\mathrm{k}$ ka ra ha? ${ }^{?}-\mathrm{t}-\mathrm{n}$ ra $]$ a

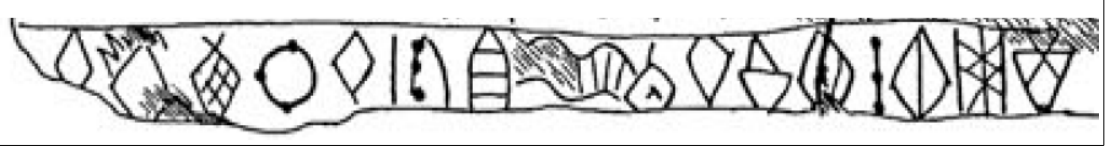

[ Puzur-Sušinak temt aúm/wa ter?] ku'-ku?-k| Šinpišu a[ki]r |

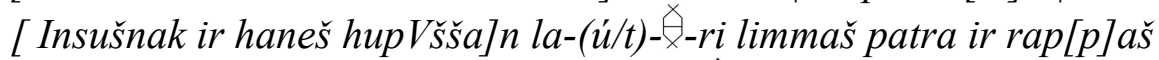

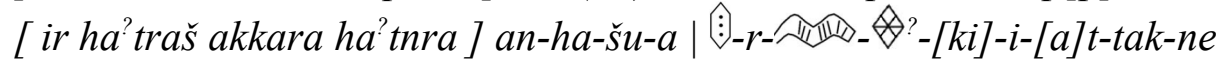

Une fois complété par comparaison avec $\mathrm{F}$ et $\mathrm{G}$, le texte $\mathrm{H}$ est le plus complet des trois. On note une variante entre $\mathrm{G}$ et $\mathrm{H}$ au milieu de la première ligne : $\left(?-k u^{?}-k u^{?}-k\right)_{G} /(?-s ̌ i k)_{H}$.

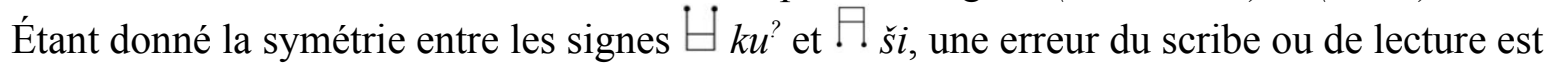
possible.

\section{$\mathrm{H}$}

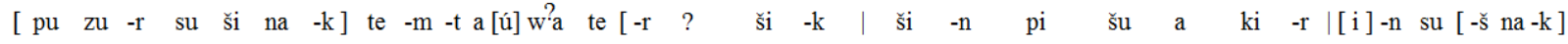
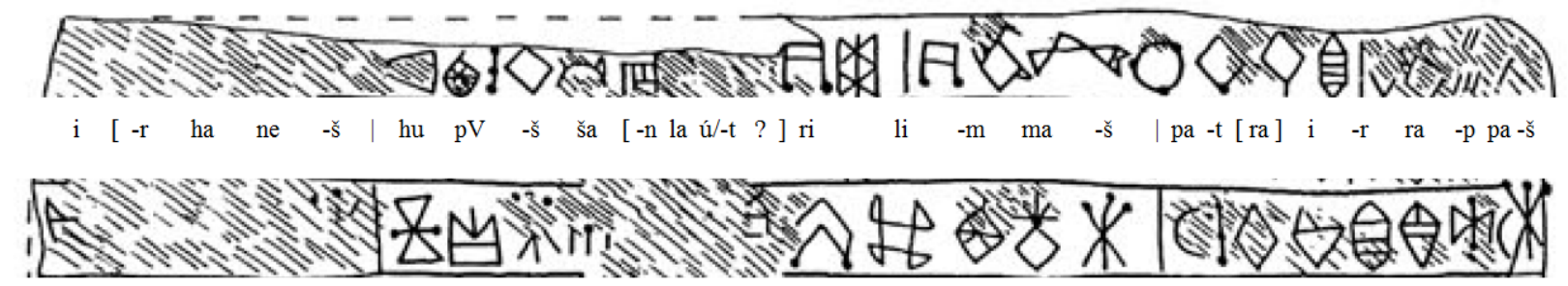

$\mathrm{i}\left[\begin{array}{ll}-\mathrm{r} & \text { ] } \\ \mathrm{ha}\end{array}\right.$

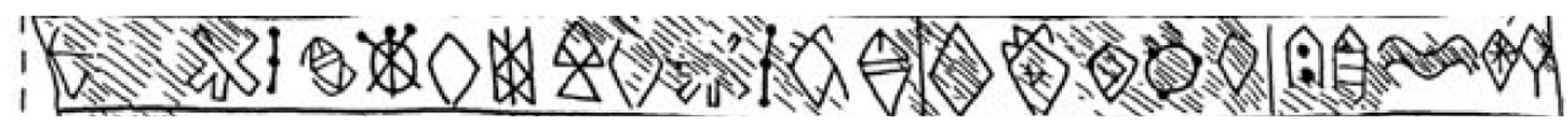

i a $\quad-t$ ta $\quad-k \quad$ ne

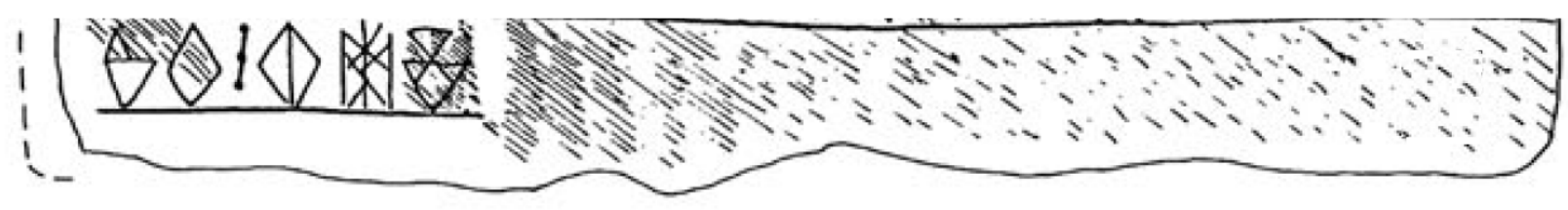


[ Puzur-Sušinak] temt a[ú]m/wa te[r? ]-šik | Šinpišu akir | [I]nsu[šnak] i[r haneš] | hupV̌šsa[n la-(ú/t)-文]-ri limmaš | pat[ra] ir rappaš

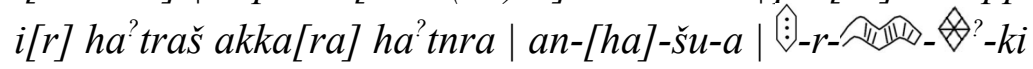
i-at-tak-ne

On peut compiler les trois textes avec leurs variantes ainsi, en nommant ce texte reconstitué FGH (les lettres en indice repèrent les variantes) :

Puzur-Sušinak temt aúm/wa ter $\mid\left\{\left(?-k u^{?}-k u^{?}-k\right)_{G} /(?-5 ̌ \text { šk })_{H} \mid \text { Šinpišu akir }\right\}_{G H} \mid$ Insušnak ir haneš $\mid$

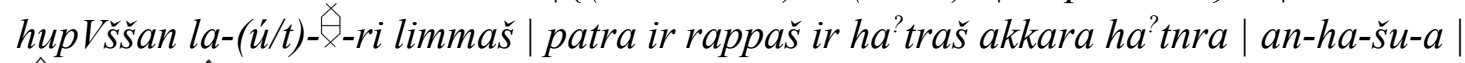
(

Ce texte est à mettre en parallèle avec le texte akkadien trouvé sur d'autres marches d'escalier [André\&Salvini 1989:65], en tous cas pour la titulature (Puzur-Inšušinak puissant roi d'Awan, fils de Šimbiišhuk) et la structure générale du texte.

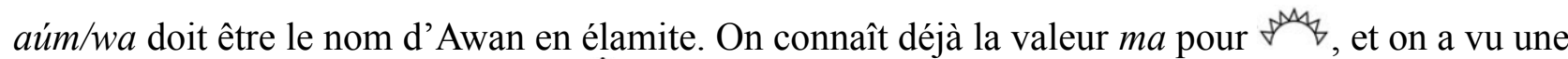
alternance $\mathrm{m} / \mathrm{w}^{24}$ dans les valeurs de $\diamond:$ we/me/ma. Il est donc possible d'avoir la même alternance pour $\stackrel{5}{\circ a}$ : $w a$.

ter : « bienveillant » comme dans A, ici en parallèle de l'akkadien dannu « puissant ».

$\left(?-k u^{?}-k u^{?}-k\right)_{G} /(?-s ̌ s i k)_{H}$ : il n'y a aucun parallèle pour cette expression.

Šinpišu akir : « fils de Šinpišu », comme dans A, avec un suffixe de 3sg. On note ici l'absence de -h final dans le nom de Šinpišu.

Insušnak ir haneš : « Insušinak l'aime ». On trouve aussi cette formule dans la titulature de ŠilhakInšušinak I ([EKI 54 §2], [EKI p. 97, note 2]) : likame Insušnak ir hanišri, qu'il faut probablement traduire « le sukkal qu'Insušnak aime ».

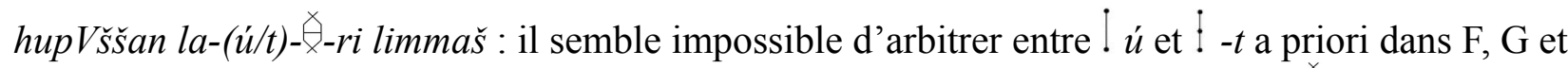

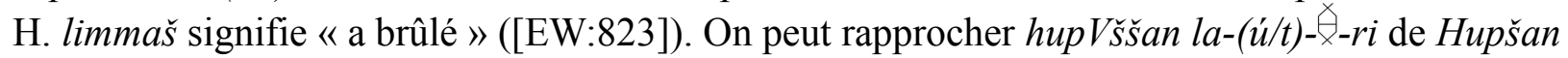
lam-lir ([Henkelman 2008:255-258]). Dans cette expression, si le - $m$ de lam est un suffixe de la classe des inanimés comme Henkelman le propose, il a pu prendre la forme -t (lat) à une époque ancienne (voir [Grillot-Susini 2008:29,42] avec l'exemple de murut/murun). On aurait donc hupV̌̌šan lat-liri en posant ${ }_{\varnothing}=$ li. Doit-on comprendre que c'est un lieu (ou une personne ?) que Puzur-Sušinak a brûlé et puni, comme la suite du texte le laisse penser?

patra ir rappaš ir ha? traš : à comparer à l'expression répétée à plusieurs reprises dans [EKI 54 §30] : li-im-ma-ak ku-ra-ak ba-at-pu-up ra-ap-ba-ak «brûlés, rôtis ?, à mes pieds punis » (voir $p a-$ at-pu-up dans [EW:112]).

patra ir rappaš pourrait donc signifier « à son pied (il) l'a puni » (ir se rapportant à hupV̌̌šan latliri).

ir ha? traš resterait dans le même champ sémantique. Il n'y a aucune occurrence de ha'tra dans le corpus connu actuellement. 
akkara ha? tnra : « quiconque déchirerait (=effacerait?) », voir ak-qa-ra dans [EW:42], ha-at-an-ra et ha-at-ta-an-ra dans [EW:582].

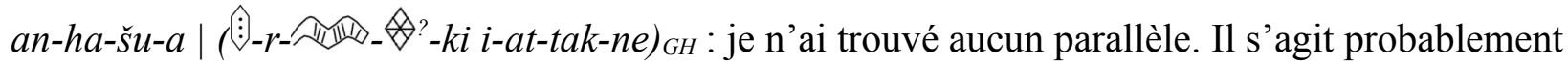
d'une malédiction portant sur celui qui effacerait l'inscription. Le dernier mot est sans doute une forme verbale comparable aux formes en -ni des malédictions d'autres textes royaux, par exemple [EKI $16 \mathrm{IV}, \mathrm{VI}]$.

Sans être une traduction du texte akkadien, la structure est la même : la titulature du roi, suivie d'une de ses actions, puis d'une malédiction.

\section{Textes de Suse : le texte $M$}

Nous pouvons appliquer les valeurs connues des caractères pour tenter de lire le texte $\mathrm{M}$ [MDP 26:XII] :

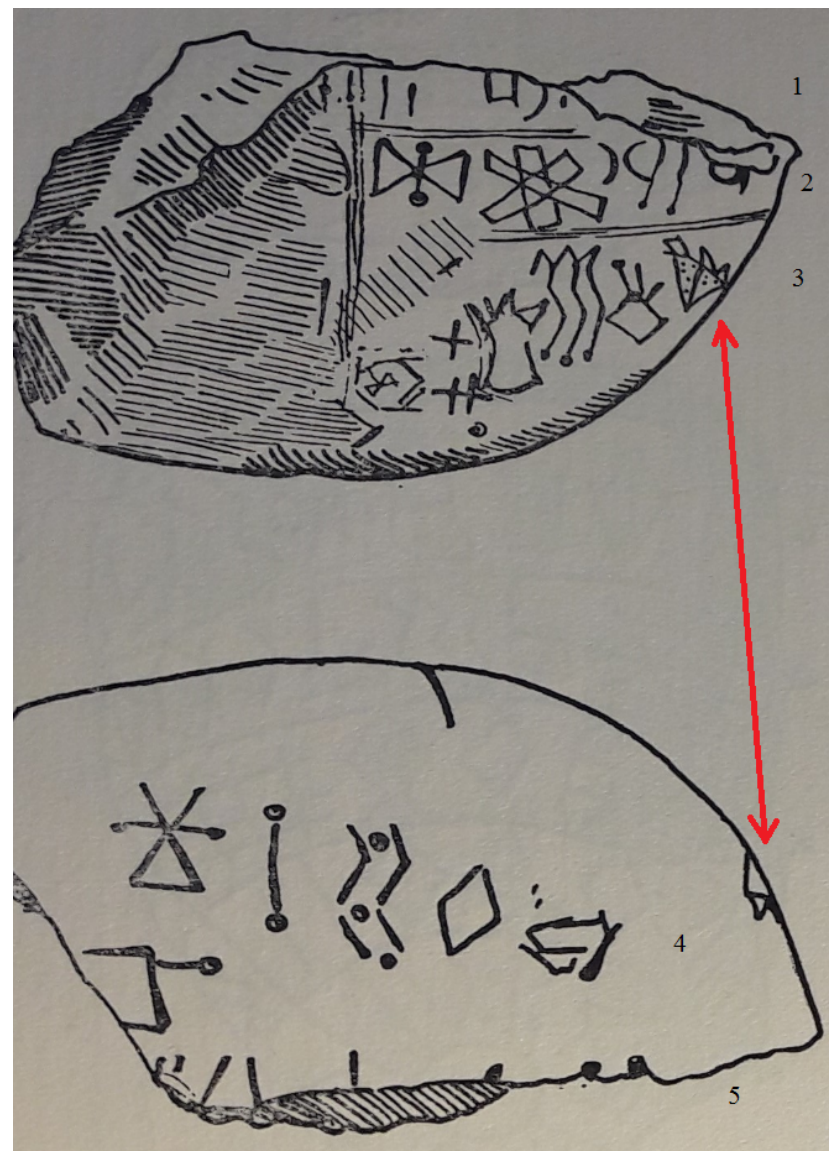

Figure 11 : le texte M [MDP 26:XII]. La flèche rouge indique un signe de l'obvers partiellement visible sur le revers.

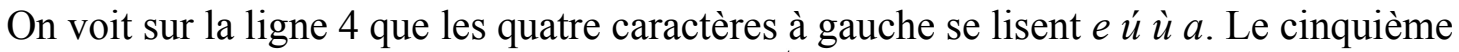
caractère n'est pas très lisible mais pourrait bien être $\theta i$. Dans ce cas, la ligne 4 correspondrait exactement à la liste de nos voyelles pour l'élamite linéaire. 
La ligne 3 ressemble à la liste de signes suivante :

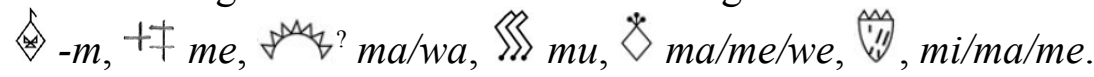

En faisant des hypothèses raisonnables sur la valeur de certains signes, cette liste correspond à la liste des voyelles de la ligne 4 , précédées à chaque fois de la consonne $m$, sauf pour le signe le plus à gauche dépourvu de voyelle. Les hypothèses à faire sont les suivantes :

- + 千 serait une variante de ;

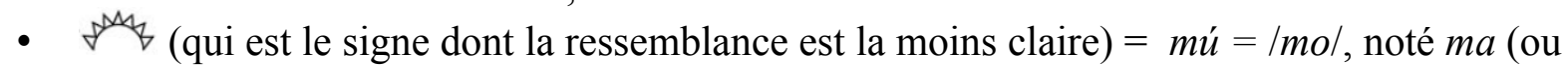
$w a$ après $\dot{u}=/ o /$ ) en élamite cunéiforme (adapté du cunéiforme akkadien, langue dépourvue de $/ 0 /)$.

- $\mathbb{S}=m \grave{u}=/ m u /$ (on le trouve dans les mots Šimut dans D et $m u h d u$ dans H', et peut-être muht dans K' si est une variante de ce signe ; dans ce cas on trouverait aussi muht dans la ligne $8 \mathrm{de} \mathrm{W}$ ).

- $\quad \nabla=m a$. C'est en effet sa valeur en début ou en milieu de mot (manip dans Z, limmaš dans F). La voyelle $a$ a pu devenir moins claire et se réduire en $e$ en syllabe finale ouverte dans likame.

- $\left(\begin{array}{ll}1 \\ \text { III }\end{array}=m i\right.$. Ce signe n'est connu que dans un mot présent dans $\mathrm{Y}, \mathrm{Z}$ et $\mathrm{K}$ ', et rendu te-im-ma, te-e-me et te-e-mi en élamite cunéiforme par les scribes. L'hésitation entre plusieurs valeurs est sans doute due à la position en syllabe finale ouverte. La valeur $m i$ est une des possibilités, et serait donc sa valeur réelle.

Après l'analyse des lignes 3 et 4 , on peut affirmer que $M$ est un texte scolaire en élamite linéaire. Cela est confirmé par la forme lenticulaire de l'objet, beaucoup de tablettes scolaires retrouvées à Suse ayant elles aussi la forme d'une lentille (voir [MDP 27:I]). Contrairement à tous les exercices connus en cunéiforme, $\mathrm{M}$ consiste apparemment en une liste phonologique de signes : liste des voyelles, liste des signes commençant par une consonne donnée.

La ligne 2 est problématique : pour ce qui en est lisible, on trouve, dans l'ordre :

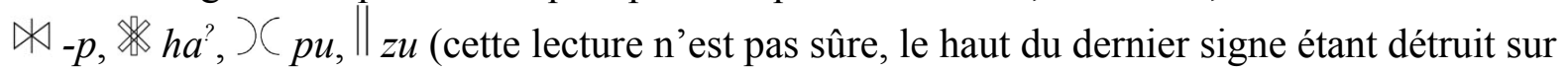
l'inscription).

Le premier et le troisième signes de cette liste sont cohérents avec les observations précédentes (on aurait alors $)(=p u=/ p o /)$.

Le deuxième signe, ${ }^{3}$ auquel nous avons attribué la valeur hypothétique $h a^{\text {? }}$, aurait en fait la valeur $p e^{?}$. Voir l'encadré « Valeur de $\$$ 》.

Le texte $M$ est important pour la compréhension de l'élamite linéaire et il serait souhaitable que des photographies en soient publiées pour approfondir son étude.

Valeur de

Mettre un tableau avec toutes les occurrences et interprétations dans les 2 cas

- $\quad h a^{?} \check{s} u$ « cèdre » dans A par pešu, ce qui est envisageable, l'interprétation de la fin de A étant plutôt hypothétique ;

- ha? tnra « déchirerait » dans F, G, H par petnra, ce qui est plus délicat, car hatanra est bien attesté et peut s'interpréter comme « effacerait» dans le contexte. On peut proposer que petnra serait rattaché à la même base que peti « ennemi » et l'interpréter dans le sens de « commettre une malveillance, une agression ». 


\section{Textes de Suse : le texte I}

Ce texte figure sur la statue de la déesse assise, dite « statue de Narundi ».

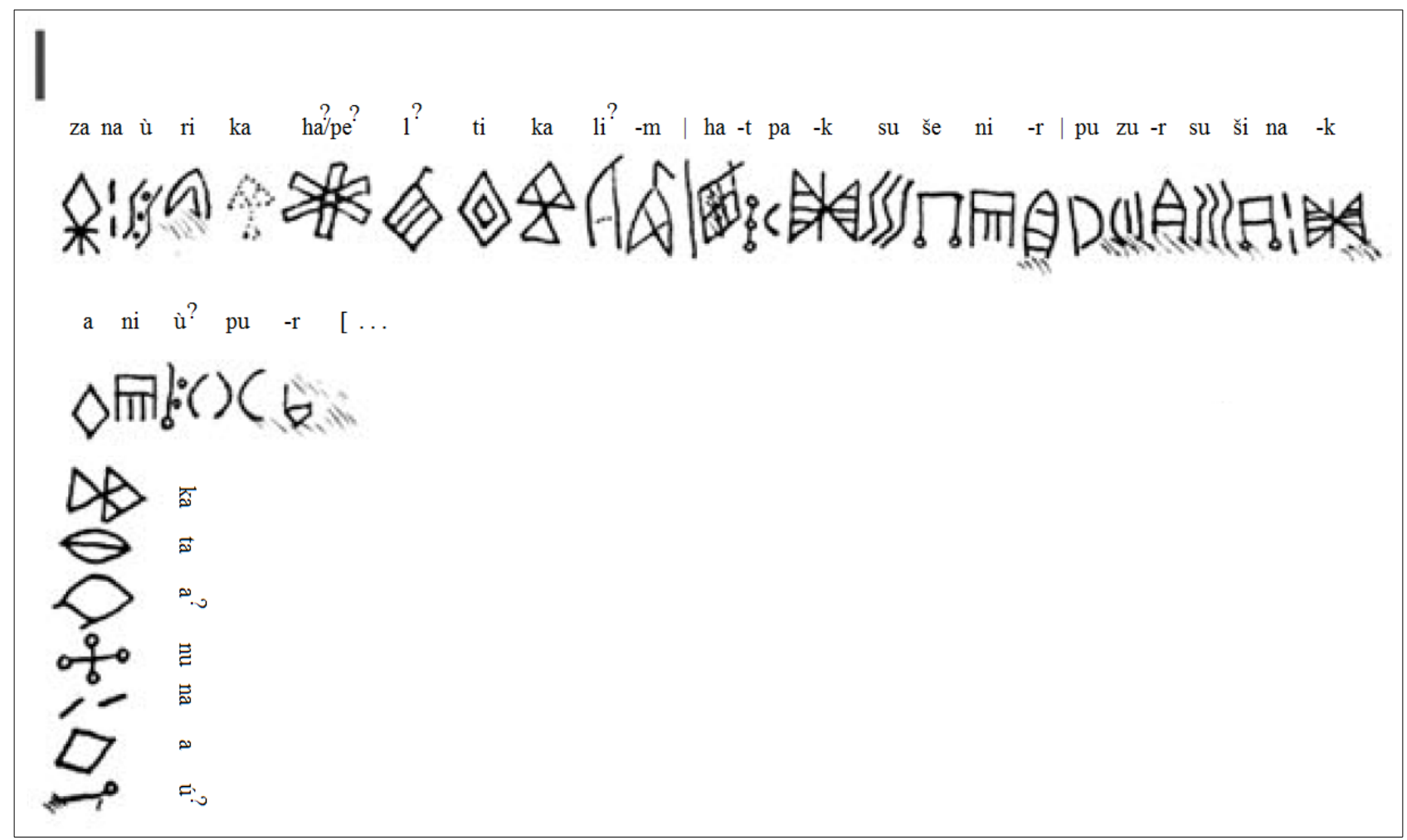

zana ù-ri-ka ha? /pe?-l’-ti-ka li’ m | hatpak sušenir | Puzur-Sušinak ani $\grave{u}^{\prime}-p u-r-[\ldots$

ka-ta-a-nu-na-a-ú?-[ ...

On reconnaît le mot zana « dame », cohérent avec la représentation féminine, et une titulature courte : « l'ensi de Suse Puzur-Sušinak ».

On peut interpréter $\grave{u}$-ri comme :

- un possessif de la première personne, mais ce n'est pas cohérent avec la troisième personne utilisée par le souverain dans sušenir ;

- un mot à rapprocher de ú-ri-pu-pi, ur-pu-up-pi « les prédécesseurs » dans [EW:1246-1248] et de urip « les anciens » dans [MDP 53:103], l'expression zana ùri signifiant alors « la Dame d'antan ».

Dans le cas où $=p e$, on pourrait lire le nom de la déesse Pelti. Dans [Vallat 2002:139], il est proposé de reconnaître Bēlti dans une dédicace d'Untaš-Napiriša pour le temple de « Innana, la Dame d'antan ».

Les deux occurrences de -ka après ùri et Pelti restent inexplicables (en supposant que le découpage des mots soit juste). 


\section{Textes de Suse : le texte D}

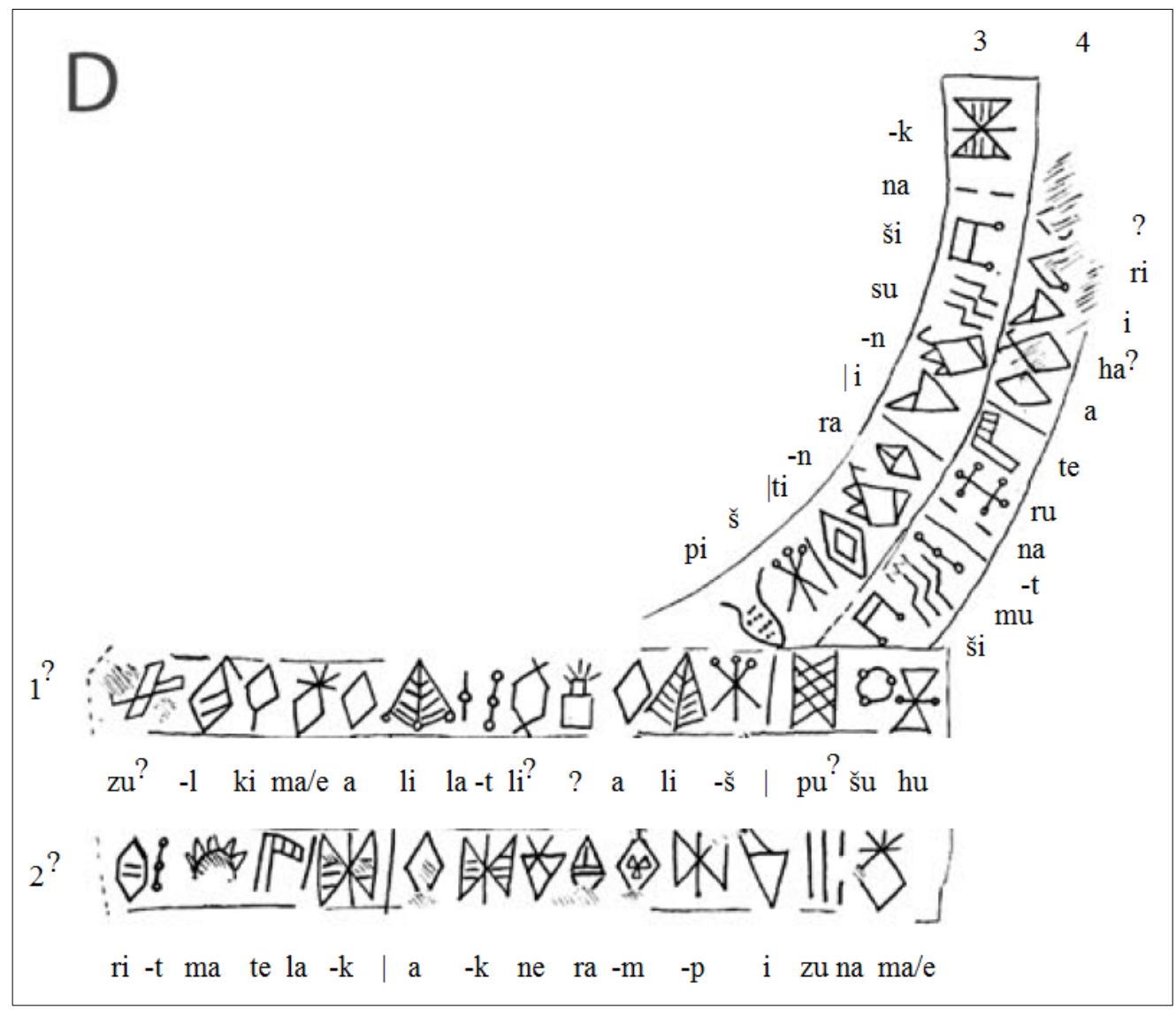

$z u^{?}-l-k i-m a / e$ ali lat-li? - 光 ališ $\mid p u^{?}-s^{\prime} u-h u$

ri-t-ma telak |a-k-ne-ra-m-p i-zu-na-ma/e

pi-š $\mid$ tinra $\mid$ Insušinak

Šimut | Narute | a-ha? i-ri [ ...

Il est difficile de se faire une idée du sens de ce texte. Il contient une suite de trois noms divins : Insušinak, Šimut et Narute (=Narunde). Pour les deux derniers, c'est leur seule mention en élamite linéaire.

On peut éventuellement reconnaître quelques mots :

- lat-li? - 光 ališ est à mettre en parallèle avec lat-liri limmaš dans F, G et H.

- On sait en effet ([Henkelman 2008:259]) que parallèlement à lam-liri, l'expression lamlema a été utilisée au temps d'Untaš-Napiriša. Le signe ${ }^{*}$ pourrait donc représenter $r V$ ou $m V$.

- ališ pourrait signifier « il a purifié » (voir a-lik dans [EW:46]).

- telak signifie « offert» (voir [EW:316]).

- On retrouve peut-être tinra dans le texte $\mathrm{S}$. 


\section{Le texte $A^{\prime}$}

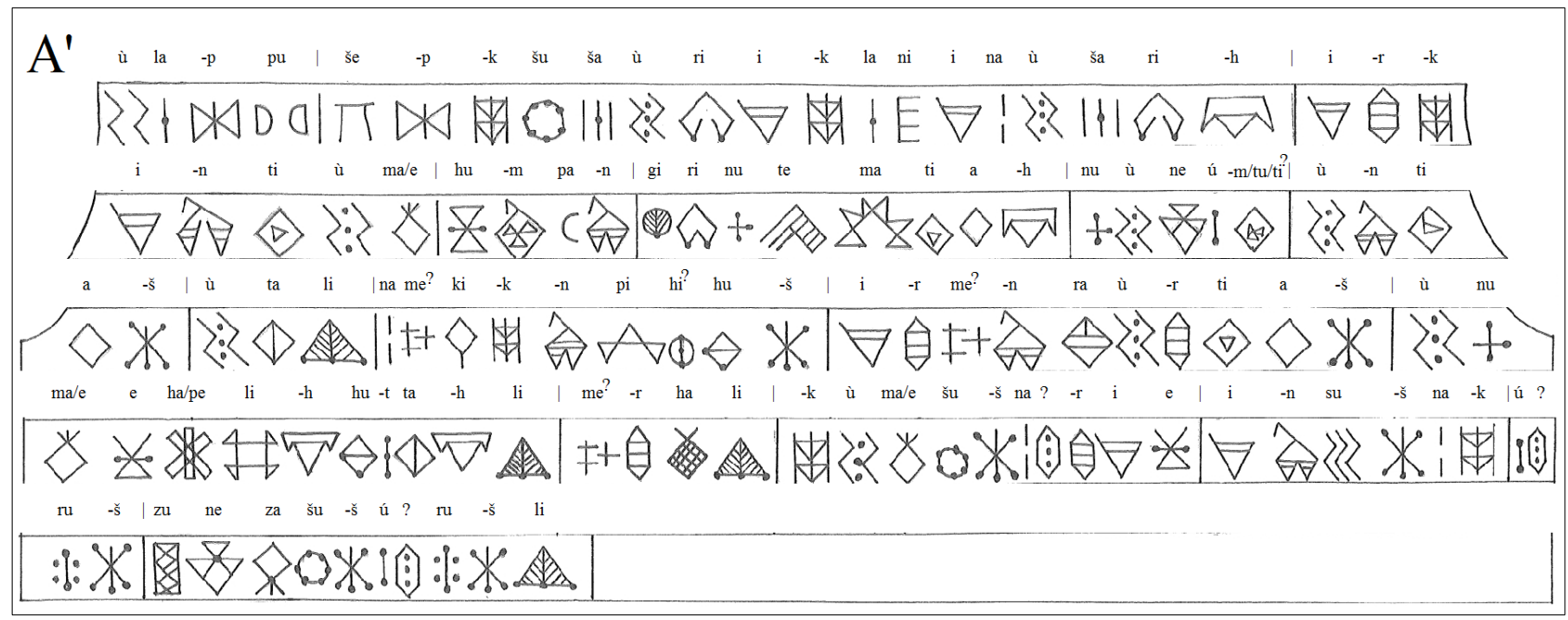

ù Lappu | ̌̌epk šsu šša-ùri-ik lani ù šarih | irk-

-inti-ù-ma/e | Humban | giri nu-te-ma tiah $\mid$ nu-ù-ne-ú-m? $/$ tu $^{?} / t^{226} \mid \grave{u} n t i-$

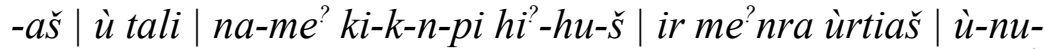

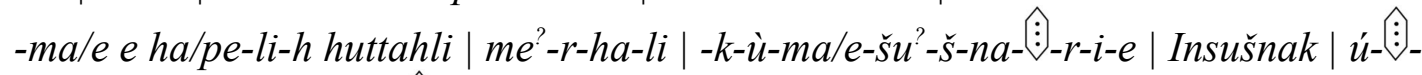

-ru-šs $\mid z u-n e-z a-\check{s} u$ ? $-\check{s} u$ -

Le signe $\hat{:}$ revient trois fois à la fin du texte, toujours suivi de la consonne $r$, de même que dans FGH. Il est probable que ces quatre occurrences reflètent une racine $\hat{\vdots}-r$. C'est une racine verbale car, dans $\dot{u} \hat{\vdots}-r u-\check{s}$ et $u^{\prime} \hat{\vdots}-r u-\check{s}$ - $l i$, elle est suivie de désinences verbales et précédée du pronom personnel objet $u$.

Dans [EW], le seul verbe conjugué correspondant au schéma $X-r u-u \check{s} / X-r u-i \check{s}$ est $t u_{4}-r u-u \check{s} / t u_{4}-r u-i \check{s}$, qui signifie « parle ! » ou « il parle, il dit ».

Je propose donc de considérer que $\hat{\vdots}=t u_{4}$.

On peut attribuer ce texte à une période proche de Puzur-Sušinak à cause de l'utilisation du signe $\left.\right|_{|l|}$ pour noter $-k$ et du titre šepk qu'on ne retrouve que dans les inscriptions de Puzur-Sušinak.

Le souverain est ici nommé Lappu, qui peut être un nom akkadien, où labbu sinifie « lion ». Sa titulature est šepk šuša-ùri (en supposant que se lise bien šu). ùri peut s'interpréter soit comme une partie du nom du territoire de Lappu, soit comme le possessif « mon », soit comme l'adjectif « ancien » (voir urip « les anciens » dans [MDP 53:103]).

Le passage irk-inti-ù-ma/e | Humban | giri nu-te-ma tiah | est à mettre en parallèle avec la formule récurrente dans les textes dits « des takkime » : ir-ki-in-ti-ú-me-ma ${ }^{d}<u n$ ou plusieurs dieux $>i$ dunih/i sima tah. L'écriture en élamite linéaire incite à revoir le découpage de irkinti en irk-inti. La formule giri nu-te-ma tiah est l'équivalent de nu giri-ma tah qu'on retrouve sur les gunagi des sukkalmah.

25 Il est possible que le signe présent dans A' se lise hi (ou même soit un nouveau signe), en effet sa graphie se situe à mi-chemin entre $O_{s} u$ et ${ }_{h i}$.

26 Le signe est mal écrit; il y a plusieurs lectures possibles. 
De la même façon que tiah est l'équivalent de tah, ùntiaš doit être celui de untaš « aidemoi » ([EW:1238]), et ùrtiaš celui de urtaš (non attesté).

La formule $n u-\grave{u}-n e-u ́-m^{?} / t u$ ? $/ t i^{?}$ peut être l'équivalent du nu ú tenti de l'époque des sukkalmah.

\section{Le texte W}

Cette inscription présente seulement quelques courts passages pouvant être mis en parallèle avec les autres textes. Sa structure est elle aussi unique : le texte est se compose de quatre parties, les trois premières contenant des répétitions de séquences. Aucun séparateur de mot n'est présent. La structure se voit mieux en réarrageant le texte sans tenir compte des sauts de ligne d'origine (les sauts proposés dans la dernière partie du texte sont plus hypothétiques) :

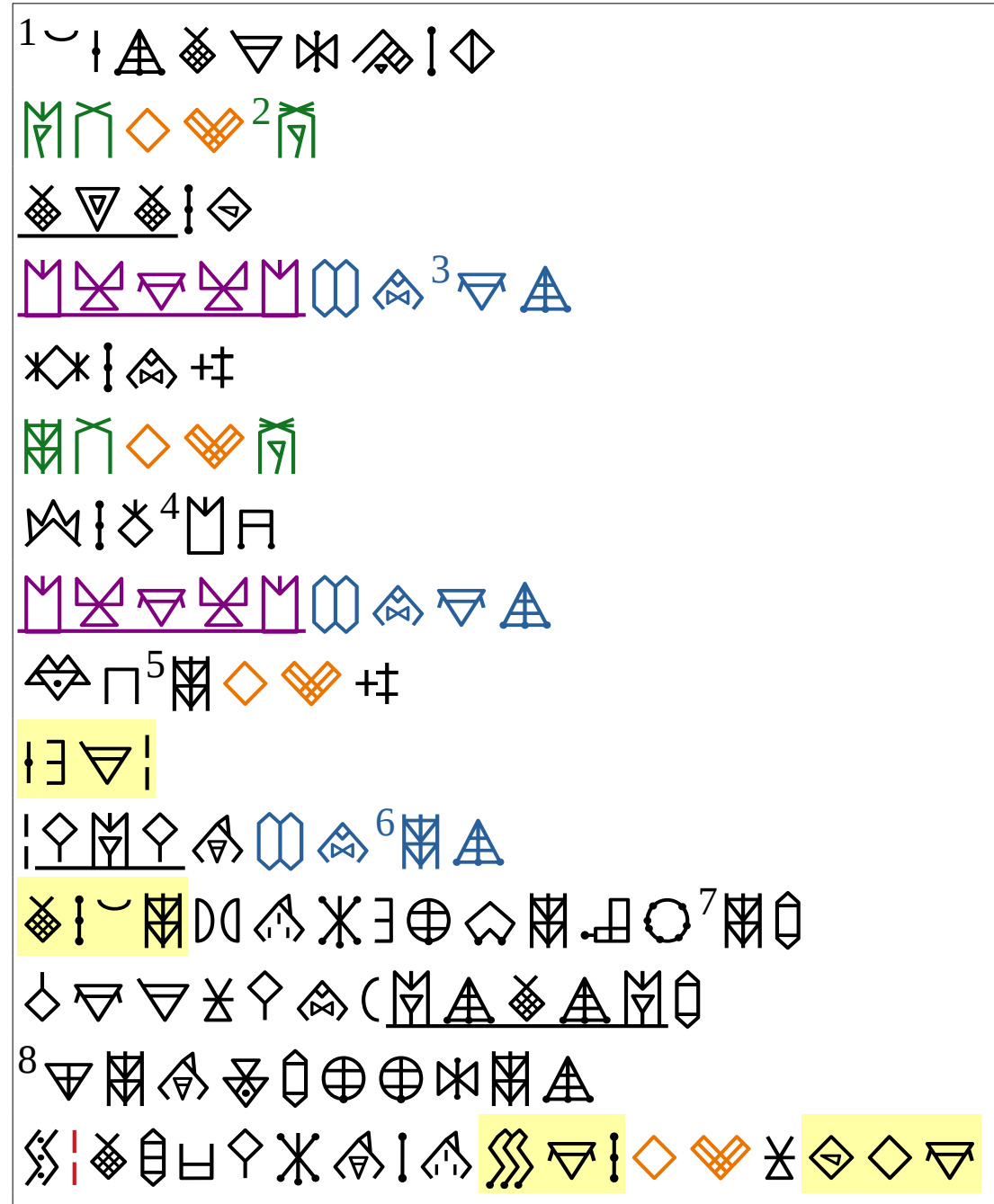

Légende

- Chiffres : début de ligne sur l'original

- Caractères en couleur : séquences identiques (ou quasi-identiques)

- Surlignage : correspondances avec des mots connus

- Soulignement : palindromes 


\section{$\underline{\text { Notes }}$}

- Il est possible que et 7 命 soient en réalité le même signe.

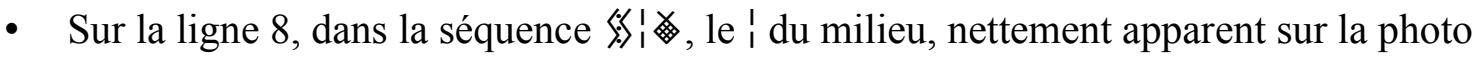
de CDLI (https://cdli.ucla.edu/search/archival view.php?ObjectID=P332981), a été omis dans [Mäder et al. 2017].

Les séquences identifiables laniina, hatpak et tiah, ainsi que la forme des signes, poussent à identifier la langue comme de l'élamite et à dater l'objet des environs du règne de Puzur-Sušinak. Cependant l'absence d'autres mots identifiables comme de l'élamite, et la présence de palindromes totalement absents des autres textes poussent à la méfiance. On peut envisager que $\mathrm{W}$ soit un texte dans une autre langue avec des inclusions d'élamite, un texte élamite relevant d'un genre nouveau, ou, comme argumente [Mäder et al. 2017], un faux.

Pour mémoire, voici les citations qu'on peut trouver concernant l'authenticité de W :

« there can be no doubt that this silver is ancient » [Scott 2011:46]

« some archaeologists still have doubts about the veracity of the inscription and feel that the inscription could have been added later » [Scott 2011:46]

« there can be no doubt whatsoever that this bucket is authentic » [Scott 2014:75]

« Nach dem momentanen Kenntnisstand ist es angebracht, die Inschrift ${ }^{\text {Phoe }}$ W vorerst als nicht authentisch zu betrachten» [Mäder et al. 2017:43] 


\section{Conclusion: proposition de syllabaire élamite linéaire}

\begin{tabular}{|c|c|c|c|c|}
\hline$\grave{u}$ & $\theta_{i}$ & $\forall_{e}$ & $\nabla_{a}$ & \multirow{3}{*}{$+-p^{28}$} \\
\hline$D O_{p u,} u^{? 27}$ & \multicolumn{2}{|c|}{ 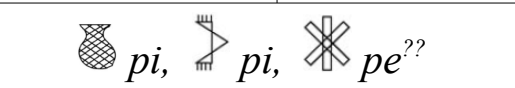 } & $C_{b a}$ & \\
\hline \multicolumn{4}{|c|}{$\mathbb{W}=w / p V(V=e / a / u)$} & \\
\hline$\otimes t u / d u, \quad \hat{\vdots}_{t u_{4}} ?$ & $\Delta \vartheta_{t i}$ & $\nabla t e^{29}, \mathbb{N l}^{m} t e$ & $\downarrow_{t a / d a}$ & $\vdots-t$ \\
\hline$\forall k u^{?}, \quad{ }_{k} u^{230}$ & \multicolumn{2}{|c|}{\rangle$_{k i,} \otimes g i$} & $\Delta_{q a}$ & 舟 $-k^{31}$ \\
\hline$O_{\check{s} u},{ }_{\check{s} u}$ & $\cap_{s ̌ i}$ & Пše & $\|$ H. $\check{s} a$ & $\mathbb{X}_{-\check{s}}$ \\
\hline 图 $\mathrm{zu} / \mathrm{su},\langle\backslash\rangle_{\mathrm{su}} / \mathrm{su}$ & \multicolumn{2}{|c|}{$\hat{i}_{s i}$} & $\hat{A}_{z a}$, 而 $s a^{? 30}$ & $\triangleleft_{-z}\left(/ s^{2} /\right)$ \\
\hline \multirow[t]{2}{*}{ 敞 $m u^{32}$} & 戍 $\mathrm{mi} / \mathrm{ma} / \mathrm{me}$ & 为 & $y^{2} \mathrm{ma} w a$ & \multirow{2}{*}{$\hat{\otimes}_{-m}$} \\
\hline & & \multicolumn{2}{|c|}{$\otimes_{m a / m e / w e}$} & \\
\hline \multirow[t]{2}{*}{$\therefore n u$} & ${ }^{1 \pi n t 133} n i$ & $\bigotimes_{n i(/ n e /)}$ & ina & $\|^{n}-n$ \\
\hline & \multicolumn{2}{|c|}{ 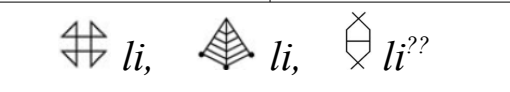 } & † la & 金 $-l$ \\
\hline$\because{ }^{34} r u$ & \multicolumn{2}{|c|}{$\hat{A}_{r i,} \hat{\vartheta}_{r i}$} & $\otimes_{r a^{35}}$ & $\hat{\theta}_{-r}$ \\
\hline$\nabla_{h u,} \nabla_{h u}$ & \multicolumn{2}{|c|}{$\bigcirc_{h i,} \quad \triangle h i^{230}$} & ${ }_{h a}, a^{? ?}$ & 网-h, \\
\hline
\end{tabular}

Figure 12 : équivalences entre élamite linéaire et valeurs de l'élamite cunéiforme

27 Uniquement attesté à Suse.

28 Attesté sur dans Z. Variante graphique plus ancienne $\nVdash$ à Suse.

29 La syllabe notée par $\nabla$ est probablement distincte de celle notée par $\|^{\| m}$.

30 On a soit (1)

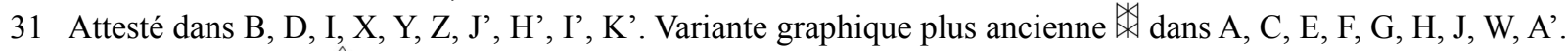

32 Variante graphique ?

33 Variante graphique $\exists$ dans A' et W.

34 Attesté dans D, Y, Z. Variante graphique $\because \because$ dans K et A'. Variante graphique (ou phonétique) IIII dans X, I', J'.

35 Attesté dans D, S, F, G, H, Q, A', F', H'. Variante graphique $\hat{\nabla}$ dans $Y$ et $Z$ ? 


\section{Remarques finales}

Au terme de ce travail, le syllabaire est quasiment complet. Un certain nombre de valeurs ne sont pas certaines et sont indiquées par des points d'interrogation. Il subsiste une alternative entre deux valeurs pour $\triangle$ et 3 .

Le système d'écriture ne comporte a priori aucun déterminatif. Quelques logogrammes sont peut-être présents parmi les signes non déchiffrés, mais l'essentiel des textes est écrit phonétiquement.

On trouve pour l'élamite un système phonologique à cinq voyelles, peut-être six si on considère que les valeurs te et me sont surchargées. Sur le plan typologique, c'est le nombre le plus courant de voyelles dans les langues du monde ([Maddieson 2013]), ce qui est plutôt rassurant. Les deux voyelles $\dot{u}$ et $\grave{u}$ ont probablement les valeurs /o/ et /u/. Vu l'absence de /o/ en akkadien, Il est

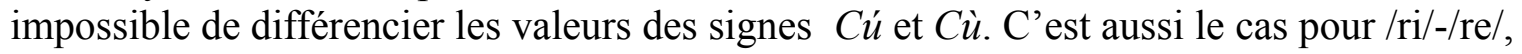
/hi/-/he/, /li/-/le/ et /ki/-/ke/ qui sont tous transcrits $C i$.

L'absence de distinction entre consonnes voisées et sourdes (réf nécessaire) est confirmée par le déchiffrement.

La surcharge de signes dans les séries $m / w$ et $p$, de même que les deux signes pour la valeur $-h$, peuvent s'expliquer par l'existence d'un /f/ en élamite. Cette consonne étant absente en akkadien, les scribes ont donc utilisé les signes cunéiformes des séries $p, w$ et $h$, qui sont les consonnes les plus proches phonétiquement.

Finalement, l'élamite linéaire paraît bien mieux adapté que le cunéiforme à l'écriture de l'élamite, et était dédié à l'écriture de cette langue sous le règne de Puzur-Sušinak et sans doute depuis une époque plus ancienne. 


\section{Bibliographie :}

[Alvarez-Mon 2019]: Alvarez-Mon, Javier. "Puzur-Inšušinak, the last king of Akkad? Text and image reconsidered." Elam and its Neighbors: Recent Research and New Perspectives. Verlag Franzbecker, 2019.

[André \& Salvini 1989] : André, Béatrice, and Mirjo Salvini. "Réflexions sur Puzur-Inšušinak." Iranica Antiqua 24 (1989): 53-72.

[Assyrian Dict 10]: Civil, M., I. J. Gelb, and A. L. Oppenheim. "The Assyrian Dictionary of the Oriental Institute of the University of Chicago, vol. 10 (M, part 1)." Oriental Institute, JJ Augustin, Chicago-Gliickstadt (1977).

[Desset 2018] : Desset, François. "Nine Linear Elamite Texts Inscribed on Silver " Gunagi " Vessels (x, Y, Z, F', H', I', J', K' and L'): New Data on Linear Elamite Writing and the History of the Sukkalmah Dynasty." Iran. 56.2 (2018): 105-143. Print.

[EKI] : König, Friedrich W. Die Elamischen Königsinschriften. Graz: E. Weidner, 1965. Print.

[EW] : Hinz, Walther, and Heidemarie Koch. Elamisches Wörterbuch: In 2 Teilen. Berlin: Reimer, 1987. Print.

[Gelb \& Kienast 1990]: Gelb, Ignace J, and Burkhart Kienast. Die Altakkadischen Königsinschriften Des Dritten Jahrtausends V. Chr. Stuttgart: F. Steiner, 1990. Print.

[Grillot-Susini 2008]: Grillot-Susini, Françoise. L'élamite: Éléments De Grammaire. Paris: Geuthner, 2008. Print.

[Henkelman 2008] : Henkelman, Wouter FM. "The other gods who are." Studies in Elamite-Iranian acculturation based on the persepolis fortification texts (2008).

[Hinz et al. 1969]: Hinz, Walther, Rykle Borger, and Gerd Gropp. Altiranische Funde Und Forschungen. Berlin: de Gruyter, 1969. Print.

[Maddieson 2013] : Maddieson, Ian. "Vowel Quality Inventories." The World Atlas of Language Structures Online. Leipzig: Max Planck Institute for Evolutionary Anthropology, 2013. Available online at http://wals.info/chapter/2, Accessed on 2019-12-05.

[Mäder et al. 2017] : Mäder, Michael, et al. "Sequenzanalysen zur elamischen Strichschrift." (2017).

[MDP 11]: Scheil, Vincent, and Paul Toscanne. Textes élamites-anzanites, quatrième série. E. Leroux, 1911.

[MDP 26]: Scheil, Vincent. Textes De Comptabilité Proto-Élamites, Troisième Série. Paris: Librairie Ernest Leroux, 1935. Print.

[MDP 27]: Meer, P E. Textes Scolaires De Suse. Paris: E. Leroux, 1936. Print.

[MDP 28]: Scheil, Vincent. "Mélanges épigraphiques (Mémoires de la Mission archéologique de Perse 28)." (1939). 
[MDP 31]: de Mecquenem, Roland, and M. Rutten. Epigraphie proto-élamite: par R. de Mecquenem. Archéologie susienne, par M. Rutten. Presses universitaires de France, 1949.

[MDP 53]: Stève, Marie-Joseph. Nouveaux Mélanges Épigraphiques: Inscriptions Royales De Suse Et De La Susiane. Nice: Editions Serre, 1987. Print.

[Meriggi 1971]: Meriggi, Piero. La Scrittura Proto-Elamica, Parte Ia: La scrittura e il contenuto dei testi. Roma: Accademia nazionale dei Lincei, 1971. Print.

[Scott 2011] : Scott, David A. Ancient metals: microstructure and metallurgy. Vol. 1. Conservation Science Press, 2011.

[Scott 2014] : Scott, David A. "Metallography and microstructure of metallic artifacts." Archaeometallurgy in Global Perspective. Springer, New York, NY, 2014. 67-89.

[Sollberger \& Kupper 1971]: Sollberger, Edmond, and Jean Robert Kupper. Inscriptions royales sumériennes et akkadiennes. Éd. du Cerf, 1971.

[Steinkeller 2013]: Steinkeller, P. "Puzur-inšušinak at Susa: a Pivotal Episode of Early Elamite History Reconsidered." Mémoires De La Délégation En Perse. 58 (2013): 293-317. Print.

[Tavernier 2007]: Tavernier, Jan. "On some Elamite signs and sounds." Zeitschrift der Deutschen Morgenländischen Gesellschaft 157.2 (2007): 265-292.

[Tavernier 2010]: Tavernier, Jan. "On the sounds rendered by the s-, š-and?/z-series in Elamite." Proceedings of the 53e Rencontre Assyriologique Internationale 1 (2010): 1059-1078.

[Töyräänvuori 2016]: Töyräänvuori, Joanna. "'" I Will Set His Hand on the Sea, and His Right Hand on the River": North West Semitic Kingship and the Sea of Combat Myth: A Survey of Hebrew Poetry in Light of Ancient Near Eastern Evidence." (2016).

[Vallat 2002] : Vallat, François. "La Dame faite prisonnière à Babylone." Akkadica 123 (2002): 137144. 